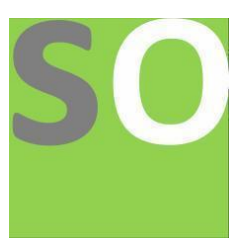

Article title: Consumption Contribution to Economy Growth: -

Authors: Ivan Sudibyo[1]

Affiliations: University of Indonesia[1]

Orcid ids: 0000-0001-6394-3813[1]

Contact e-mail: ivansudibyo14@gmail.com

License information: This work has been published open access under Creative Commons Attribution License $\mathrm{http}: / / c r e a t i v e c o m m o n s . o r g / l i c e n s e s / b y / 4.0 /$, which permits unrestricted use, distribution, and reproduction in any medium, provided the original work is properly cited. Conditions, terms of use and publishing policy can be found at https://www.scienceopen.com/.

Preprint statement: This article is a preprint and has not been peer-reviewed, under consideration and submitted to ScienceOpen Preprints for open peer review.

Links to data: https://osf.io/xbytd

Funder: -

DOI: 10.14293/S2199-1006.1.SOR-.PPVQHAO.v1

Preprint first posted online: 23 December 2021

Keywords: consumption, growth 
Consumption Contribution to Economy Growth

Ivan Sudibyo

\begin{abstract}
The analysis of the final consumption influence on the Gross Domestic Product is rarely studied. The final consumption is one important studies that follow the expenses method of GDP calculation and formation. The econometric approach gives substantial results when a longer interval is approached. The fact of econometric model is the dependence of the national economy on final consumption. Thus, the unifactorial regression model can be used to establish the influence that the value of final consumption. The Researcher make some description about final consumption in Southeast Asia+3 and US.
\end{abstract}


"GDP has recently faced unusually intense criticism with some commentators calling for it to be replaced by a more direct measure of wellbeing based on selfreports. One problem is not the concept itself, but the way it is used, and that too much is expected of it. The use of GDP as the headline measure of economic progress is particularly unfortunate, and has become more so as economies have changed and become more globally connected. I shall say something about this first, and then about how we should extend national accounts to handle distributional issues, and finally about the problem of measuring healthcare. I should also note that while I am very much in favor of the collection of self-reported measures of wellbeing, including both evaluations of wellbeing and reports of feelings, I do not think it makes sense to think of them as replacements for GDP or other measures in the national accounts. Extensive work has proved that these measures are useful, that they sometimes capture important aspects of life that are not otherwise measured, and that much can be learned from comparing them with other, more familiar, measures. I think it is unfortunate that self-reports of wellbeing are not regularly collected somewhere in the American statistical system" (Deaton, 2020).

"Again, there is nothing wrong with this situation, at least statistically, but if the news media and politicians continue to valorize GDP or per capita GDP, the concept will lose repute, undermining public confidence in the national accounts. Much better would be to focus on what is happening to people, through their levels of disposable income or consumption" (Deaton, 2020).

The analysis of the final consumption influence on the Gross Domestic Product is one of the most important studies that follow the expenses method of GDP calculation and formation. The econometric approach gives substantial results when a longer interval is approached and, one of the truths behind the econometric model is the dependence of the national economy on final consumption. Thus, the unifactorial regression model can be used to establish the influence that the value of final consumption has on the evolution of Gross Domestic Product (Anghel, et.al, 2017).

Danquah, M., et.al., (2014) find that the most robust TFP growth determinants are unobserved heterogeneity, initial GDP, consumption share, and trade openness. A split of the sample into OECD and non-OECD countries reveals some interesting findings. Danquah, M., et.al., (2014) find that initial GDP and unobserved heterogeneity are the only two robust determinants common to OECD and non-OECD countries. For the sample of OECD countries, in addition to the fixed effects and initial GDP, the results find that investment price, consumption share, trade openness, and the labor force are robustly correlated to TFP growth. With respect to non-OECD countries, the only additional variable robustly correlated with TFP growth is population density. 
Asumadu-Sarkodie, et.al. (2016) used variance decomposition and find that show that almost $19 \%$ of future fluctuations in household final consumption expenditure are due to shocks in GDP, while 13\% of future fluctuations in household final consumption expenditure are due to shocks in mortality rate. Meaning that GDP affects household final consumption expenditure more than mortality rate, fertility rate, and the food production index in the longrun in Ghana. Besides that, $6 \%$ of future fluctuations in GDP are due to shocks in household final consumption expenditure the while $5,75 \%$ of future fluctuations in GDP are due to shocks in food production index. Meaning that food production index affects GDP more than fertility rate, mortality rate in the long-run in Ghana.

Shaikh, N. A., et. al. (2015) found that consumption function in Pakistan estimates show significant, strong and positive relationship between GDP and consumption expenditure. P-values (in braces) associated with constant and GDP variable in column 5 (under $\mathrm{t}$ ) are $<5 \%$ or 0.05 so the estimates have high generalizability power. The slope of the consumption function is the rate of change in consumption due to the change in income by one unit. The function result is $\mathrm{C}=-4484.05+0.821 \mathrm{Y}$ and the Multiplier $=\frac{1}{1-M P C}=\frac{1}{1=0.821}=5.587$.

The other research state that the association between consumption, income and GDP was found significant in the all the considered panels of countries, more accentuated for the low and middle income countries. The fact that a greater level of consumption and income increases the proxy of the standard of living, but to a lower degree for the high income countries which are more proficient in investments and R\&D activities, especially in human capital (Diacon, P.E., et.al., 2015).

The other paper said that consumption and production were usually seen in a relationship of mutually beneficial causation: increased consumption is not only the result but also the cause of a greater production of wealth, since it increases labor productivity (Perrotta,C., 1997).

Research methodology and data

Researcher want to describe the consumption data in Southeast Asia +3 and US Countries. Researcher used data on a yearly basis covering the period 1960-2020 from World Bank indicators database. Based on these observations and methodological elements mentioned above, Researcher used regression method as a tool to estimate the parameters of this model. The results can be summarized as follows. The following is an illustration of the consumption of ASEAN + 3 countries and the US; 
Dependent Variable: USA_GDPUSD

Method: Least Squares

Sample (adjusted): 19702020

Included observations: 51 after adjustments

\begin{tabular}{lcccc}
\hline \hline Variable & Coefficient & Std. Error & t-Statistic & Prob. \\
\hline \hline USA_CONSM & 1.200462 & 0.005287 & 227.0640 & 0.0000 \\
C & $1.94 \mathrm{E}+11$ & $4.75 \mathrm{E}+10$ & 4.076330 & 0.0002 \\
\hline \hline R-squared & 0.999051 & Mean dependent var & $9.04 \mathrm{E}+12$ \\
Adjusted R-squared & 0.999031 & S.D. dependent var & $6.25 \mathrm{E}+12$ \\
S.E. of regression & $1.94 \mathrm{E}+11$ & Akaike info criterion & 54.86325 \\
Sum squared resid & $1.85 \mathrm{E}+24$ & Schwarz criterion & 54.93901 \\
Log likelihood & -1397.013 & Hannan-Quinn criter. & 54.89220 \\
F-statistic & 51558.04 & Durbin-Watson stat & 0.217703 \\
Prob(F-statistic) & 0.000000 & & \\
\hline \hline
\end{tabular}

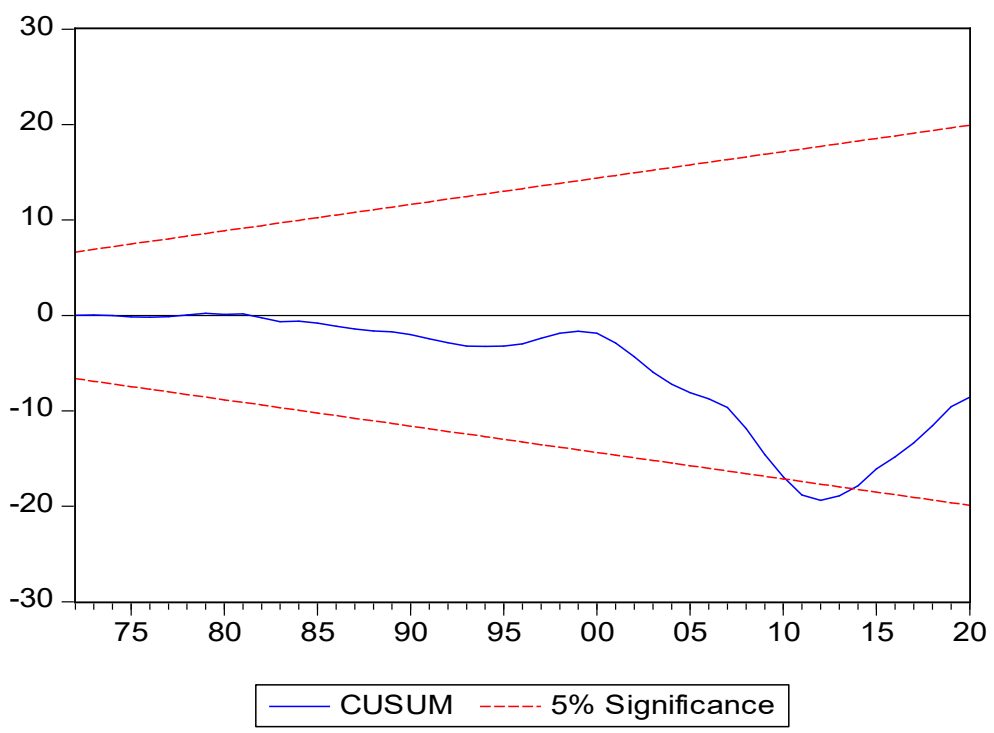


Dependent Variable: CHN_GDPUSD

Method: Least Squares

Sample (adjusted): 19602019

Included observations: 60 after adjustments

\begin{tabular}{lllll}
\hline \hline Variable & Coefficient & Std. Error & t-Statistic & Prob. \\
\hline \hline CHN_CONSM & 1.859384 & 0.011585 & 160.4990 & 0.0000 \\
C & $-1.87 \mathrm{E}+10$ & $2.85 \mathrm{E}+10$ & -0.655044 & 0.5150 \\
\hline \hline R-squared & 0.997754 & Mean dependent var & $2.37 \mathrm{E}+12$ \\
Adjusted R-squared & 0.997715 & S.D. dependent var & $3.94 \mathrm{E}+12$ \\
S.E. of regression & $1.89 \mathrm{E}+11$ & Akaike info criterion & 54.79607 \\
Sum squared resid & $2.06 \mathrm{E}+24$ & Schwarz criterion & 54.86588 \\
Log likelihood & -1641.882 & Hannan-Quinn criter. & 54.82338 \\
F-statistic & 25759.94 & Durbin-Watson stat & 0.166240 \\
Prob(F-statistic) & 0.000000 & & \\
\hline \hline
\end{tabular}

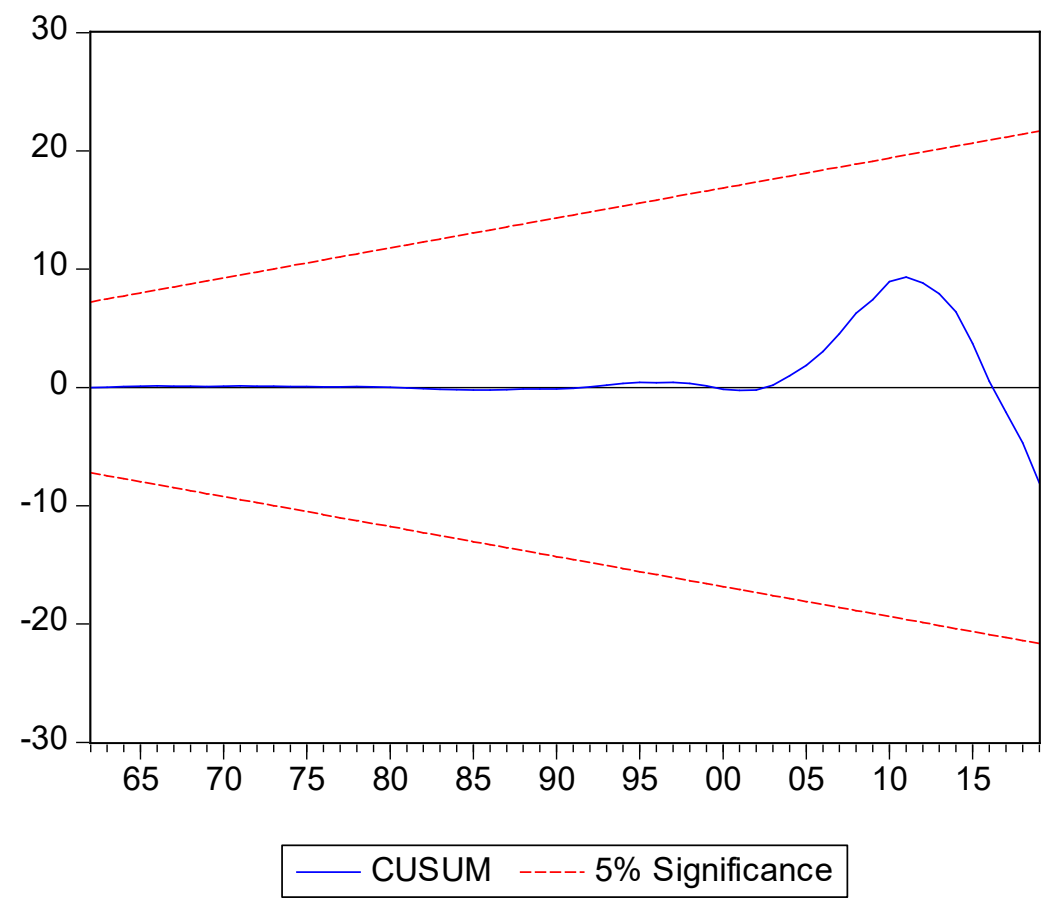


Dependent Variable: JPN_GDPUSD

Method: Least Squares

Sample (adjusted): 19702019

Included observations: 50 after adjustments

\begin{tabular}{lcccc}
\hline \hline Variable & Coefficient & Std. Error & t-Statistic & Prob. \\
\hline \hline JPN_CONSM & 1.320872 & 0.019267 & 68.55548 & 0.0000 \\
C & $1.91 \mathrm{E}+11$ & $5.39 \mathrm{E}+10$ & 3.539330 & 0.0009 \\
\hline \hline R-squared & 0.989890 & Mean dependent var & $3.36 \mathrm{E}+12$ \\
Adjusted R-squared & 0.989680 & S.D. dependent var & $1.94 \mathrm{E}+12$ \\
S.E. of regression & $1.97 \mathrm{E}+11$ & Akaike info criterion & 54.89115 \\
Sum squared resid & $1.86 \mathrm{E}+24$ & Schwarz criterion & 54.96763 \\
Log likelihood & -1370.279 & Hannan-Quinn criter. & 54.92028 \\
F-statistic & 4699.854 & Durbin-Watson stat & 0.093385 \\
Prob(F-statistic) & 0.000000 & & \\
\hline \hline
\end{tabular}

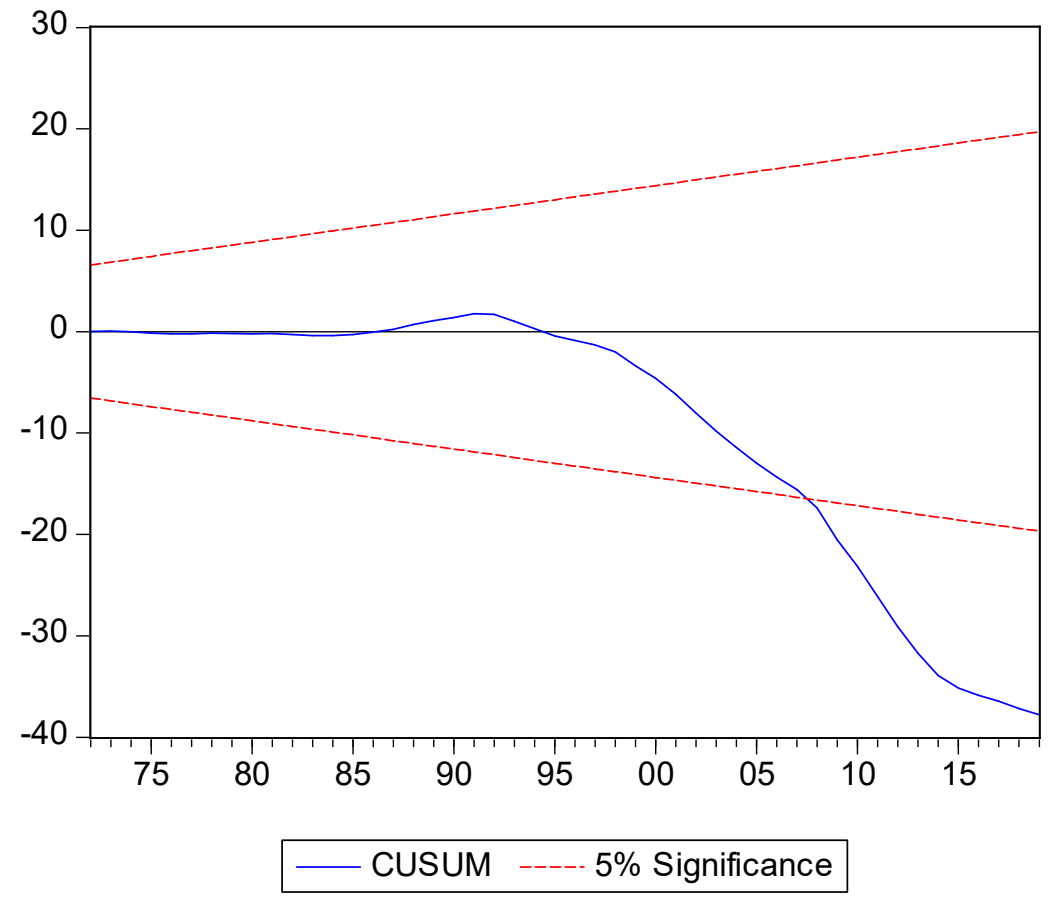


Dependent Variable: KOR_GDPUSD

Method: Least Squares

Sample: 19602020

Included observations: 61

\begin{tabular}{lllll}
\hline \hline Variable & Coefficient & Std. Error & t-Statistic & Prob. \\
\hline \hline KOR_CONSM & 1.544471 & 0.005569 & 277.3367 & 0.0000 \\
C & $-5.08 \mathrm{E}+08$ & $2.68 \mathrm{E}+09$ & -0.189480 & 0.8504 \\
\hline \hline R-squared & 0.999234 & Mean dependent var & $5.00 \mathrm{E}+11$ \\
Adjusted R-squared & 0.999221 & S.D. dependent var & $5.55 \mathrm{E}+11$ \\
S.E. of regression & $1.55 \mathrm{E}+10$ & Akaike info criterion & 49.79903 \\
Sum squared resid & $1.42 \mathrm{E}+22$ & Schwarz criterion & 49.86824 \\
Log likelihood & -1516.870 & Hannan-Quinn criter. & 49.82615 \\
F-statistic & 76915.62 & Durbin-Watson stat & 0.605002 \\
Prob(F-statistic) & 0.000000 & & \\
\hline \hline
\end{tabular}

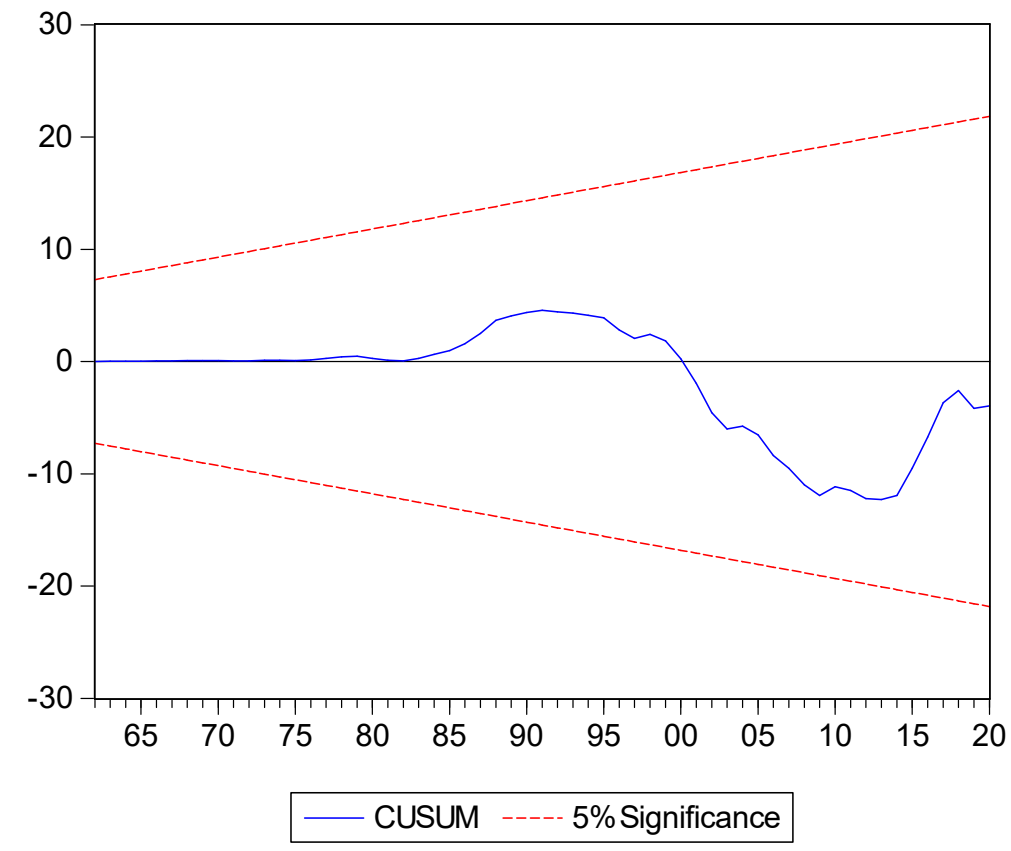


Dependent Variable: SGP_GDPUSD

Method: Least Squares

Sample: 19602020

Included observations: 61

\begin{tabular}{lllll}
\hline \hline Variable & Coefficient & Std. Error & t-Statistic & Prob. \\
\hline \hline SGP_CONSM & 2.177244 & 0.015223 & 143.0245 & 0.0000 \\
C & $-5.19 \mathrm{E}+09$ & $1.08 \mathrm{E}+09$ & -4.820480 & 0.0000 \\
\hline \hline R-squared & 0.997124 & Mean dependent var & $9.53 \mathrm{E}+10$ \\
Adjusted R-squared & 0.997075 & S.D. dependent var & $1.18 \mathrm{E}+11$ \\
S.E. of regression & $6.36 \mathrm{E}+09$ & Akaike info criterion & 48.01704 \\
Sum squared resid & $2.39 \mathrm{E}+21$ & Schwarz criterion & 48.08625 \\
Log likelihood & -1462.520 & Hannan-Quinn criter. & 48.04417 \\
F-statistic & 20456.01 & Durbin-Watson stat & 0.476363 \\
Prob(F-statistic) & 0.000000 & & \\
\hline \hline
\end{tabular}

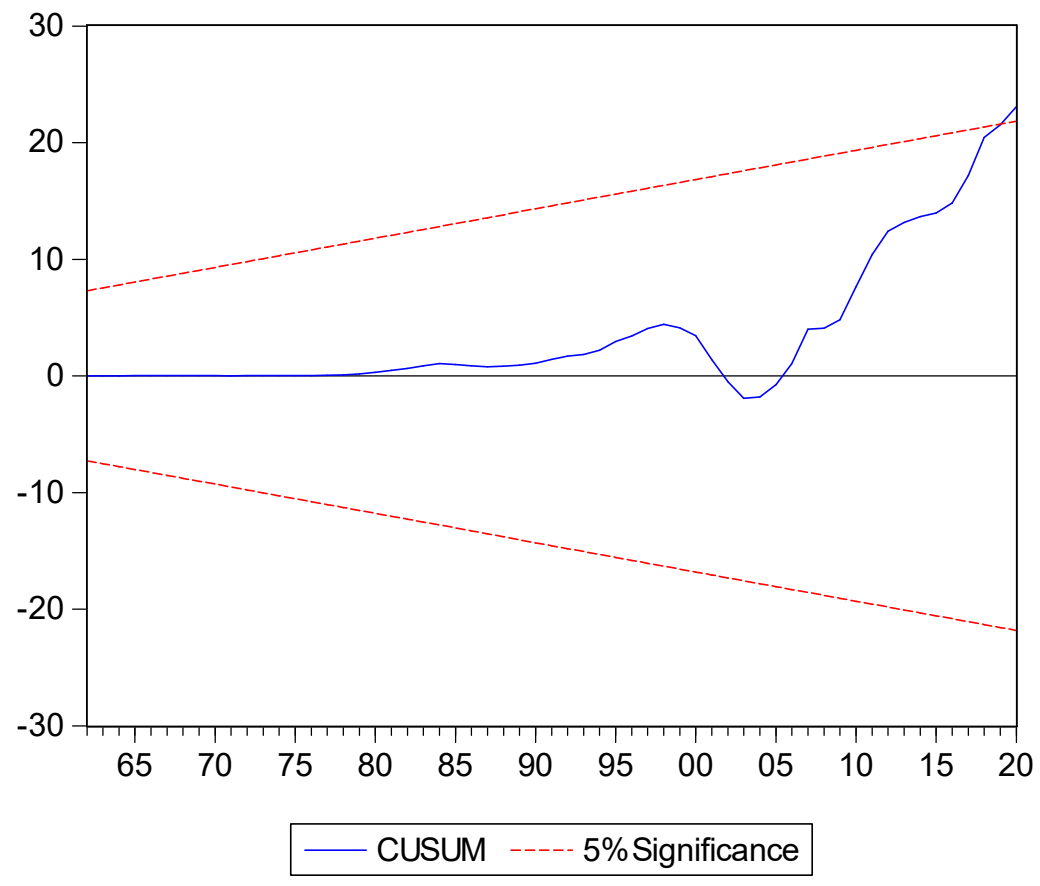


Dependent Variable: THA_GDPUSD

Method: Least Squares

Sample: 19602020

Included observations: 61

\begin{tabular}{lllll}
\hline \hline Variable & Coefficient & Std. Error & t-Statistic & Prob. \\
\hline \hline THA_CONSM & 1.481647 & 0.008185 & 181.0308 & 0.0000 \\
C & $-7.77 \mathrm{E}+08$ & $1.17 \mathrm{E}+09$ & -0.664440 & 0.5090 \\
\hline \hline R-squared & 0.998203 & Mean dependent var & $1.42 \mathrm{E}+11$ \\
Adjusted R-squared & 0.998172 & S.D. dependent var & $1.58 \mathrm{E}+11$ \\
S.E. of regression & $6.74 \mathrm{E}+09$ & Akaike info criterion & 48.13300 \\
Sum squared resid & $2.68 \mathrm{E}+21$ & Schwarz criterion & 48.20221 \\
Log likelihood & -1466.056 & Hannan-Quinn criter. & 48.16012 \\
F-statistic & 32772.15 & Durbin-Watson stat & 0.705779 \\
Prob(F-statistic) & 0.000000 & & \\
\hline \hline
\end{tabular}

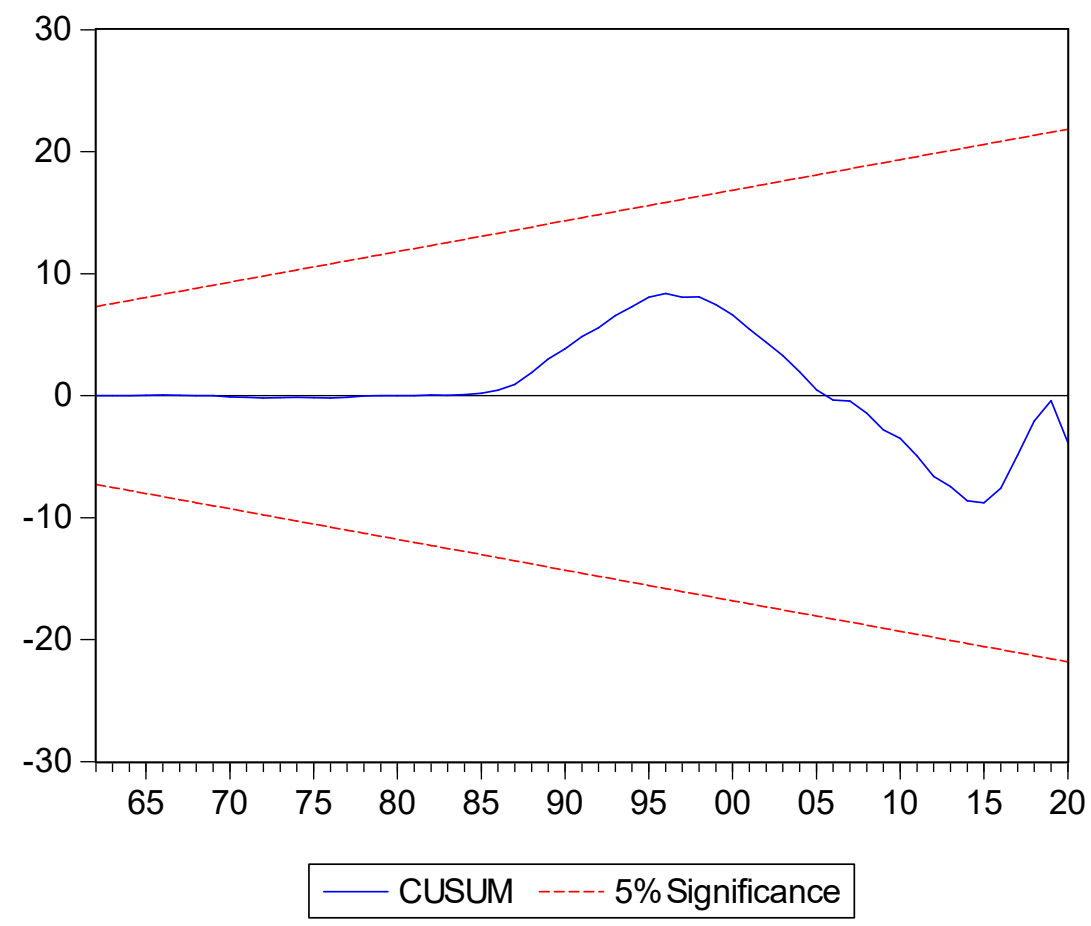


Dependent Variable: PHL_GDPUSD

Method: Least Squares

Sample: 19602020

Included observations: 61

\begin{tabular}{lllll}
\hline \hline Variable & Coefficient & Std. Error & t-Statistic & Prob. \\
\hline \hline PHL_CONSM & 1.177430 & 0.006107 & 192.8082 & 0.0000 \\
C & $1.99 \mathrm{E}+09$ & $7.34 \mathrm{E}+08$ & 2.705613 & 0.0089 \\
\hline \hline R-squared & 0.998415 & Mean dependent var & $9.37 \mathrm{E}+10$ \\
Adjusted R-squared & 0.998389 & S.D. dependent var & $1.09 \mathrm{E}+11$ \\
S.E. of regression & $4.37 \mathrm{E}+09$ & Akaike info criterion & 47.26512 \\
Sum squared resid & $1.13 \mathrm{E}+21$ & Schwarz criterion & 47.33433 \\
Log likelihood & -1439.586 & Hannan-Quinn criter. & 47.29224 \\
F-statistic & 37174.99 & Durbin-Watson stat & 0.552236 \\
Prob(F-statistic) & 0.000000 & & \\
\hline \hline
\end{tabular}

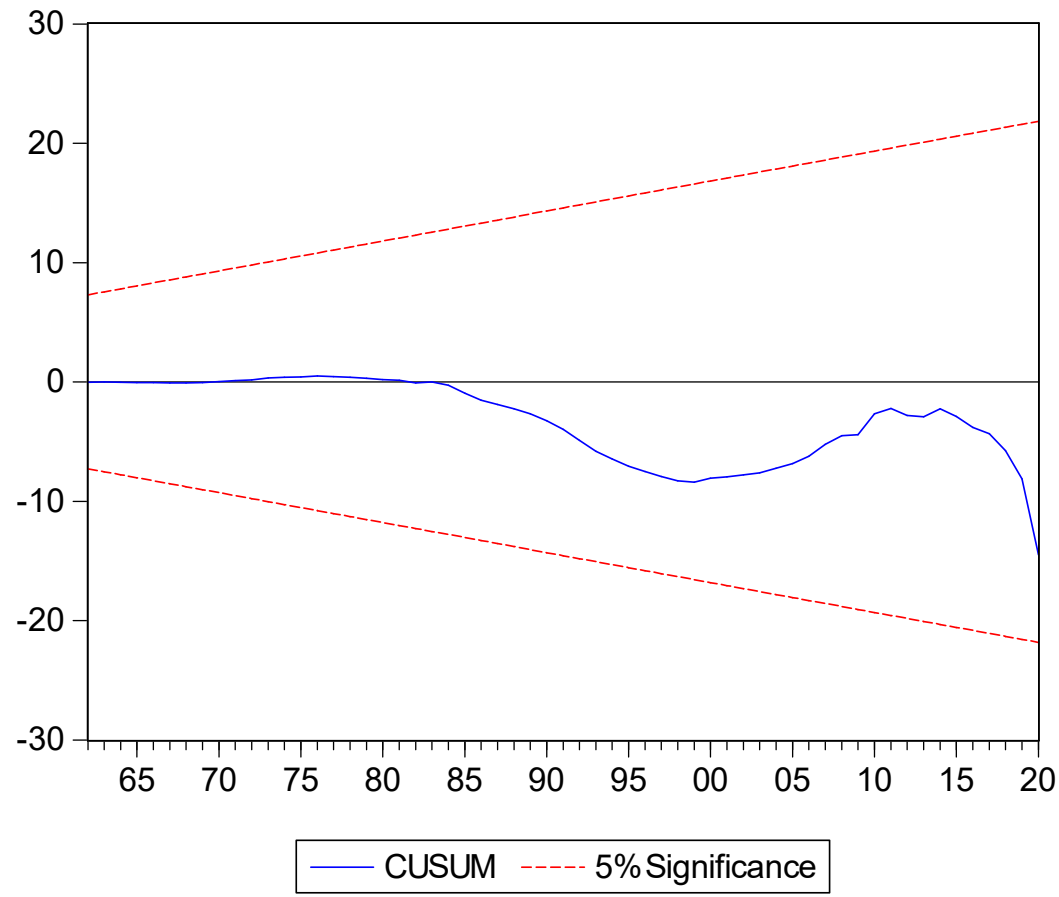


Dependent Variable: MYS_GDPUSD

Method: Least Squares

Sample: 19602020

Included observations: 61

\begin{tabular}{lllll}
\hline \hline Variable & Coefficient & Std. Error & t-Statistic & Prob. \\
\hline \hline MYS_CONSM & 1.491460 & 0.019915 & 74.89166 & 0.0000 \\
C & $5.04 \mathrm{E}+09$ & $2.00 \mathrm{E}+09$ & 2.524423 & 0.0143 \\
\hline \hline R-squared & 0.989590 & Mean dependent var & $1.01 \mathrm{E}+11$ \\
Adjusted R-squared & 0.989414 & S.D. dependent var & $1.17 \mathrm{E}+11$ \\
S.E. of regression & $1.20 \mathrm{E}+10$ & Akaike info criterion & 49.28532 \\
Sum squared resid & $8.49 \mathrm{E}+21$ & Schwarz criterion & 49.35453 \\
Log likelihood & -1501.202 & Hannan-Quinn criter. & 49.31244 \\
F-statistic & 5608.761 & Durbin-Watson stat & 0.157843 \\
Prob(F-statistic) & 0.000000 & & \\
\hline \hline
\end{tabular}

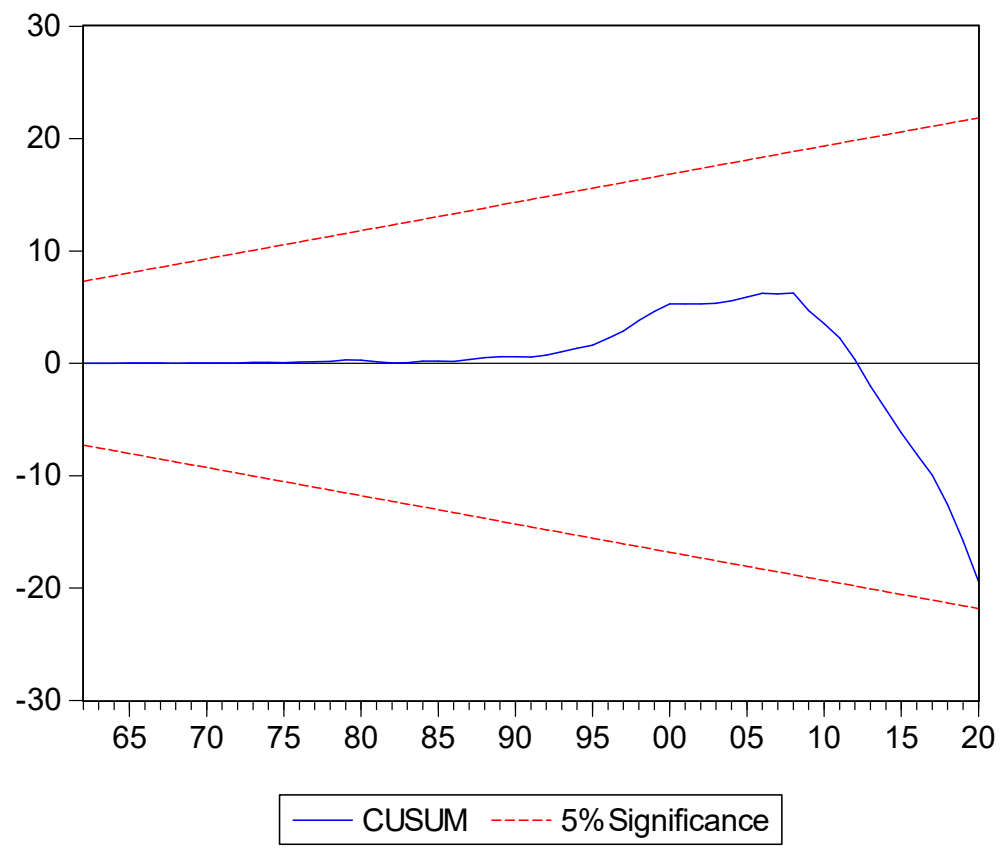


Dependent Variable: IDN_GDPUSD

Method: Least Squares

Sample (adjusted): 19672020

Included observations: 54 after adjustments

\begin{tabular}{lllll}
\hline \hline Variable & Coefficient & Std. Error & t-Statistic & Prob. \\
\hline \hline IDN_CONSM & 1.512733 & 0.006848 & 220.8882 & 0.0000 \\
C & $-1.05 \mathrm{E}+10$ & $2.12 \mathrm{E}+09$ & -4.954327 & 0.0000 \\
\hline \hline R-squared & 0.998935 & Mean dependent var & $3.01 \mathrm{E}+11$ \\
Adjusted R-squared & 0.998915 & S.D. dependent var & $3.52 \mathrm{E}+11$ \\
S.E. of regression & $1.16 \mathrm{E}+10$ & Akaike info criterion & 49.22034 \\
Sum squared resid & $6.98 \mathrm{E}+21$ & Schwarz criterion & 49.29400 \\
Log likelihood & -1326.949 & Hannan-Quinn criter. & 49.24875 \\
F-statistic & 48791.58 & Durbin-Watson stat & 0.671424 \\
Prob(F-statistic) & 0.000000 & & \\
\hline \hline
\end{tabular}

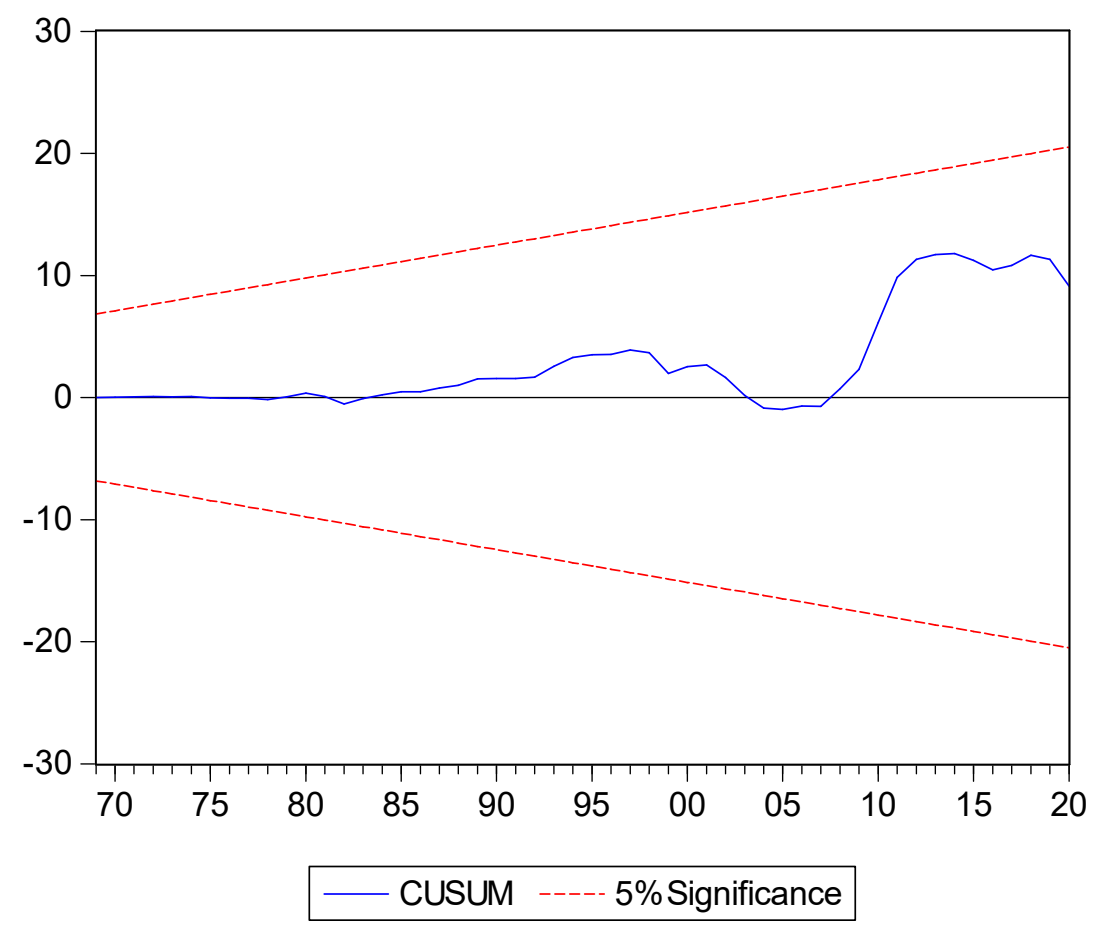


Dependent Variable: VNM_GDPUSD

Method: Least Squares

Sample (adjusted): 19892020

Included observations: 32 after adjustments

\begin{tabular}{|c|c|c|c|c|}
\hline Variable & Coefficient & Std. Error & t-Statistic & Prob. \\
\hline VNM_CONSM & 1.355078 & 0.007946 & 170.5266 & 0.0000 \\
\hline & & & & \\
\hline R-squared & 0.998969 & \multicolumn{2}{|c|}{ Mean dependent var } & $9.21 \mathrm{E}+10$ \\
\hline Adjusted R-squared & 0.998935 & \multicolumn{2}{|c|}{ S.D. dependent var } & $8.55 E+10$ \\
\hline S.E. of regression & $2.79 E+09$ & \multicolumn{2}{|c|}{ Akaike info criterion } & 46.39641 \\
\hline Sum squared resid & $2.33 \mathrm{E}+20$ & \multicolumn{2}{|c|}{ Schwarz criterion } & 46.48802 \\
\hline Log likelihood & -740.3426 & \multirow{2}{*}{\multicolumn{2}{|c|}{ Hannan-Quinn criter. }} & 46.42678 \\
\hline F-statistic & 29079.34 & & & 0.500657 \\
\hline Prob(F-statistic) & 0.000000 & \multicolumn{2}{|c|}{ Durbin-Watson stat } & \\
\hline
\end{tabular}

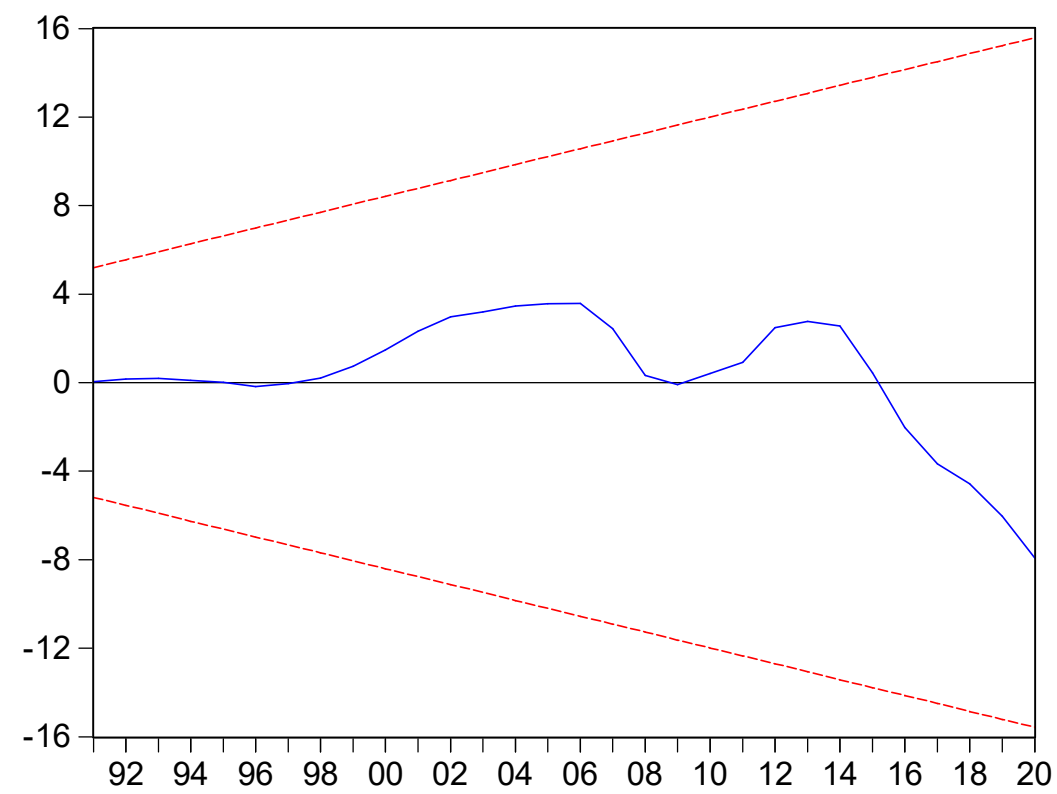

- CUSUM ----- 5\% Significance 
Dependent Variable: BRN_GDPUSD

Method: Least Squares

Sample (adjusted): 19742020

Included observations: 42 after adjustments

\begin{tabular}{lllll}
\hline \hline Variable & Coefficient & Std. Error & t-Statistic & Prob. \\
\hline \hline BRN_CONSM & 2.330328 & 0.154651 & 15.06827 & 0.0000 \\
C & $1.39 \mathrm{E}+08$ & $5.98 \mathrm{E}+08$ & 0.231988 & 0.8177 \\
\hline \hline R-squared & 0.850217 & Mean dependent var & $7.76 \mathrm{E}+09$ \\
Adjusted R-squared & 0.846472 & S.D. dependent var & $5.26 \mathrm{E}+09$ \\
S.E. of regression & $2.06 \mathrm{E}+09$ & Akaike info criterion & 45.77552 \\
Sum squared resid & $1.70 \mathrm{E}+20$ & Schwarz criterion & 45.85827 \\
Log likelihood & -959.2860 & Hannan-Quinn criter. & 45.80585 \\
F-statistic & 227.0529 & Durbin-Watson stat & 0.400873 \\
Prob(F-statistic) & 0.000000 & & \\
\hline \hline
\end{tabular}

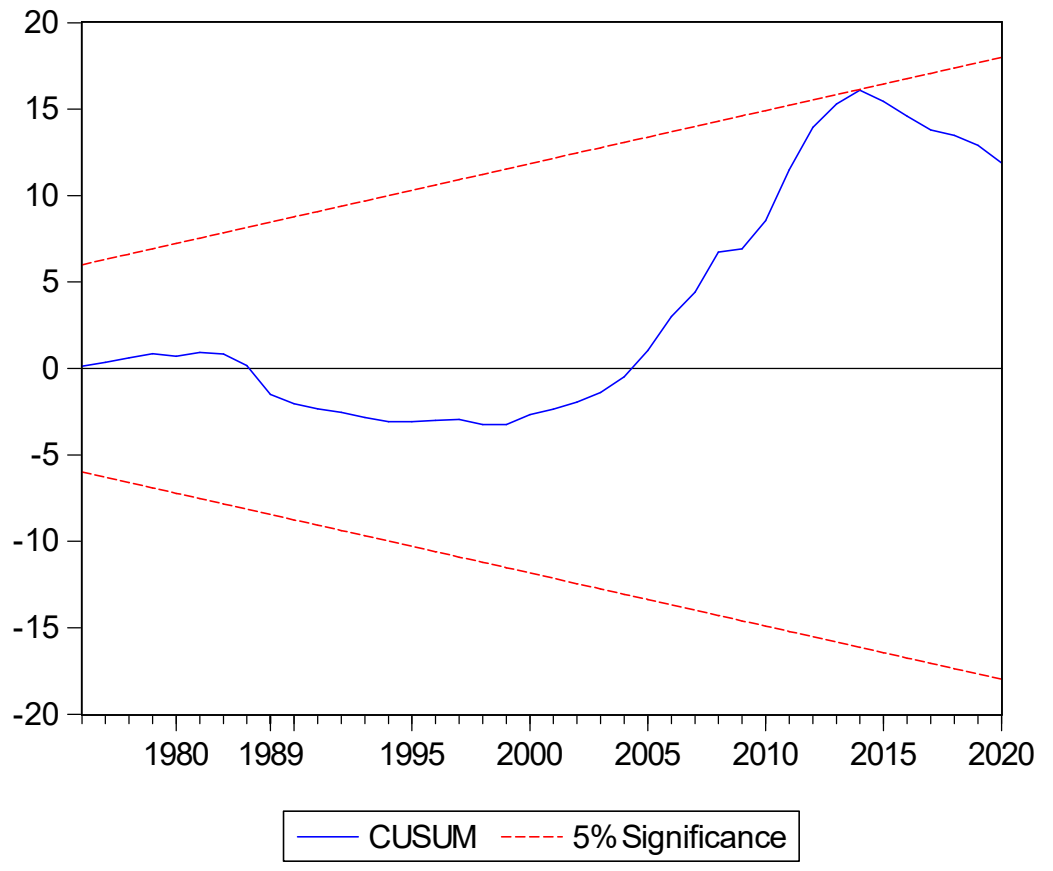


Dependent Variable: LAO_GDPUSD

Method: Least Squares

Sample (adjusted): 19842016

Included observations: 22 after adjustments

\begin{tabular}{lllll}
\hline \hline Variable & Coefficient & Std. Error & t-Statistic & Prob. \\
\hline \hline LAO_CONSM & 1.175179 & 0.022843 & 51.44525 & 0.0000 \\
C & -37716169 & $1.40 \mathrm{E}+08$ & -0.268898 & 0.7908 \\
\hline \hline R-squared & 0.992500 & Mean dependent var & $5.47 \mathrm{E}+09$ \\
Adjusted R-squared & 0.992125 & S.D. dependent var & $4.79 \mathrm{E}+09$ \\
S.E. of regression & $4.25 \mathrm{E}+08$ & Akaike info criterion & 42.65841 \\
Sum squared resid & $3.61 \mathrm{E}+18$ & Schwarz criterion & 42.75760 \\
Log likelihood & -467.2425 & Hannan-Quinn criter. & 42.68178 \\
F-statistic & 2646.614 & Durbin-Watson stat & 1.416272 \\
Prob(F-statistic) & 0.000000 & & \\
\hline \hline
\end{tabular}

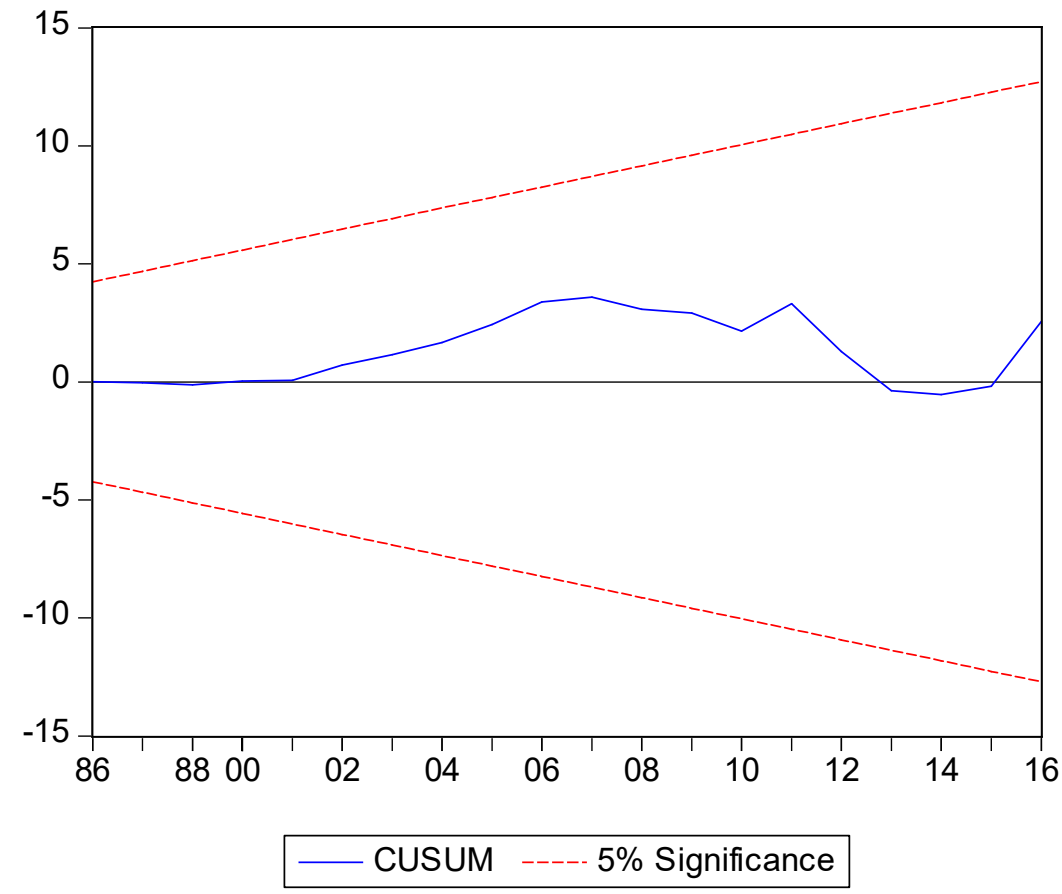


Dependent Variable: KHM_GDPUSD

Method: Least Squares

Sample: 19602020

Included observations: 39

\begin{tabular}{lllll}
\hline \hline Variable & Coefficient & Std. Error & t-Statistic & Prob. \\
\hline \hline KHM_CONSM & 1.289217 & 0.016117 & 79.99102 & 0.0000 \\
C & $-5.82 \mathrm{E}+08$ & $1.43 \mathrm{E}+08$ & -4.066270 & 0.0002 \\
\hline \hline R-squared & 0.994251 & Mean dependent var & $7.78 \mathrm{E}+09$ \\
Adjusted R-squared & 0.994095 & S.D. dependent var & $7.94 \mathrm{E}+09$ \\
S.E. of regression & $6.10 \mathrm{E}+08$ & Akaike info criterion & 43.34548 \\
Sum squared resid & $1.38 \mathrm{E}+19$ & Schwarz criterion & 43.43079 \\
Log likelihood & -843.2368 & Hannan-Quinn criter. & 43.37608 \\
F-statistic & 6398.564 & Durbin-Watson stat & 0.289010 \\
Prob(F-statistic) & 0.000000 & & \\
\hline \hline
\end{tabular}

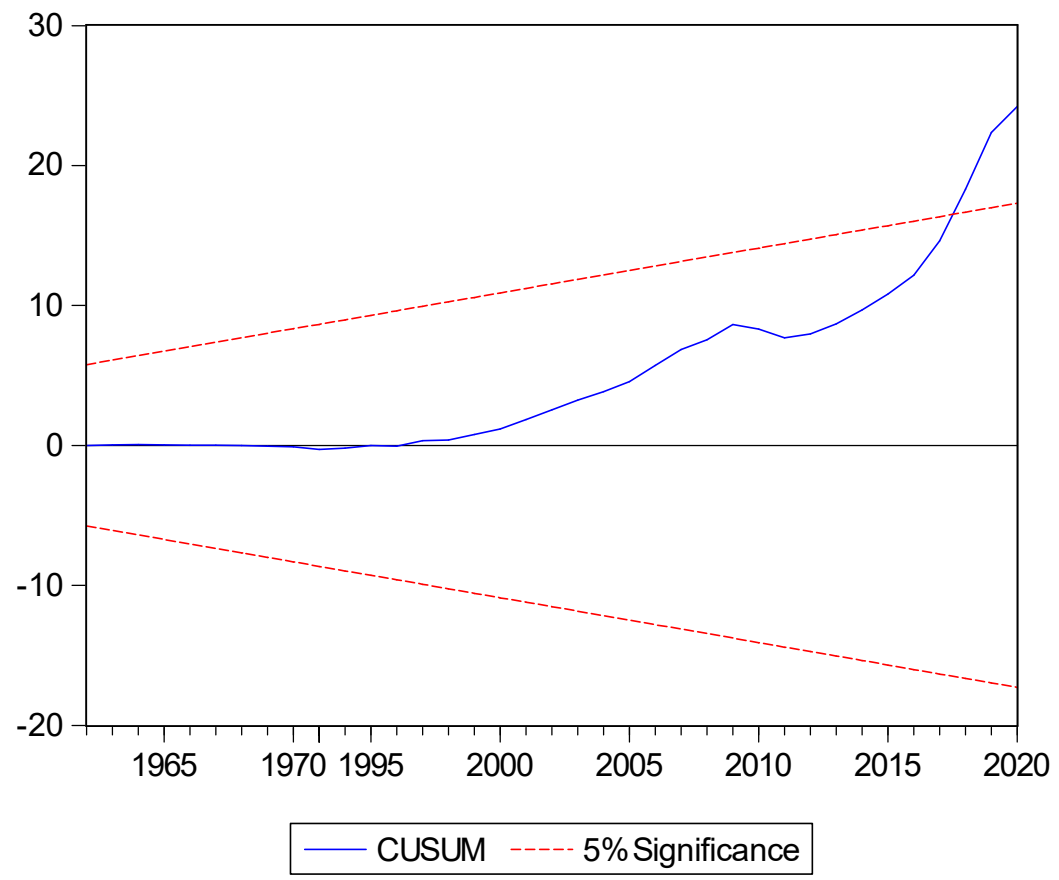


Dependent Variable: MMR_GDPUSD

Method: Least Squares

Sample (adjusted): 20082020

Included observations: 13 after adjustments

\begin{tabular}{|c|c|c|c|c|}
\hline Variable & Coefficient & Std. Error & t-Statistic & Prob. \\
\hline $\begin{array}{l}\text { MMR_CONSM } \\
\mathrm{C}\end{array}$ & $\begin{array}{l}1.457123 \\
-1.50 E+09\end{array}$ & $\begin{array}{l}0.163001 \\
6.87 E+09\end{array}$ & $\begin{array}{l}8.939337 \\
-0.217838\end{array}$ & $\begin{array}{l}0.0000 \\
0.8315\end{array}$ \\
\hline $\begin{array}{l}\text { R-squared } \\
\text { Adjusted R-squared } \\
\text { S.E. of regression } \\
\text { Sum squared resid } \\
\text { Log likelihood } \\
\text { F-statistic } \\
\text { Prob(F-statistic) }\end{array}$ & $\begin{array}{l}0.879004 \\
0.868004 \\
4.73 \mathrm{E}+09 \\
2.46 \mathrm{E}+20 \\
-306.9540 \\
79.91174 \\
0.000002\end{array}$ & \multicolumn{2}{|c|}{$\begin{array}{l}\text { Mean dependent var } \\
\text { S.D. dependent var } \\
\text { Akaike info criterion } \\
\text { Schwarz criterion } \\
\text { Hannan-Quinn criter. } \\
\text { Durbin-Watson stat }\end{array}$} & $\begin{array}{l}5.88 \mathrm{E}+10 \\
1.30 \mathrm{E}+10 \\
47.53138 \\
47.61830 \\
47.51352 \\
0.850615\end{array}$ \\
\hline
\end{tabular}

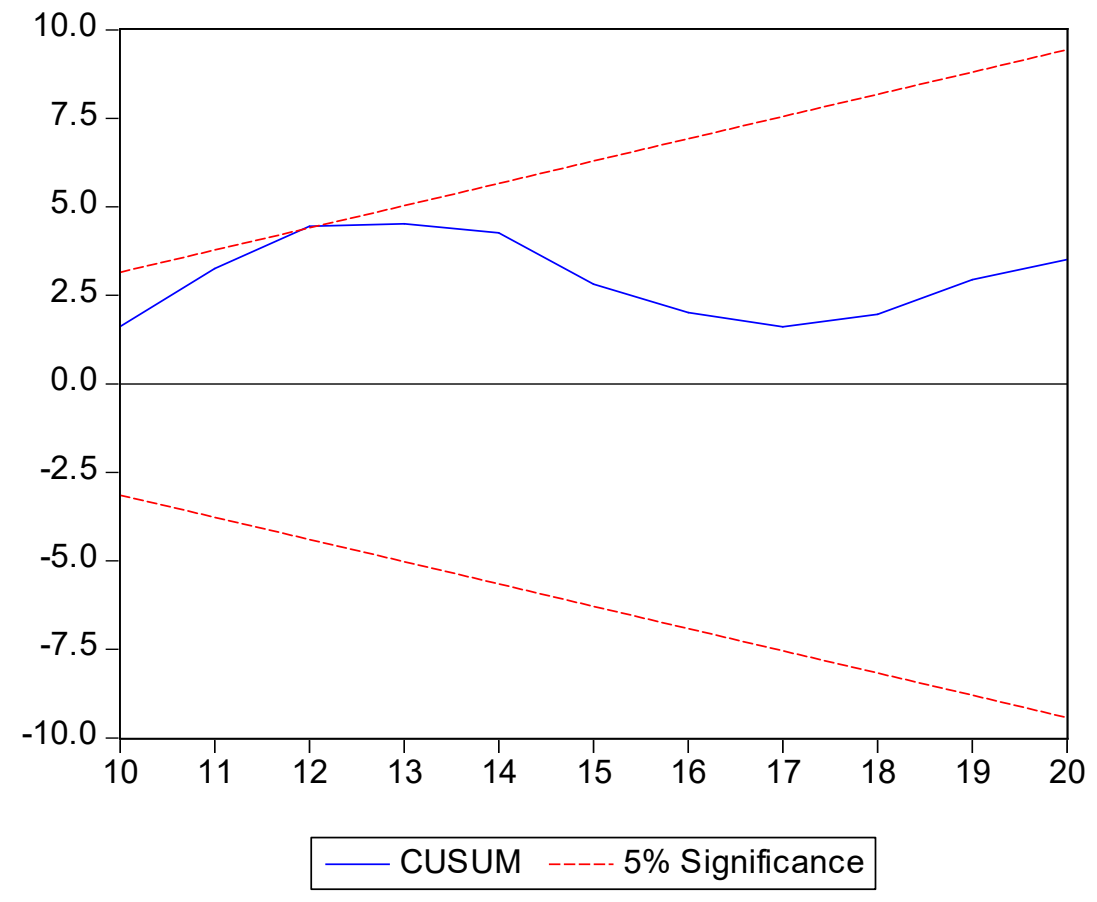




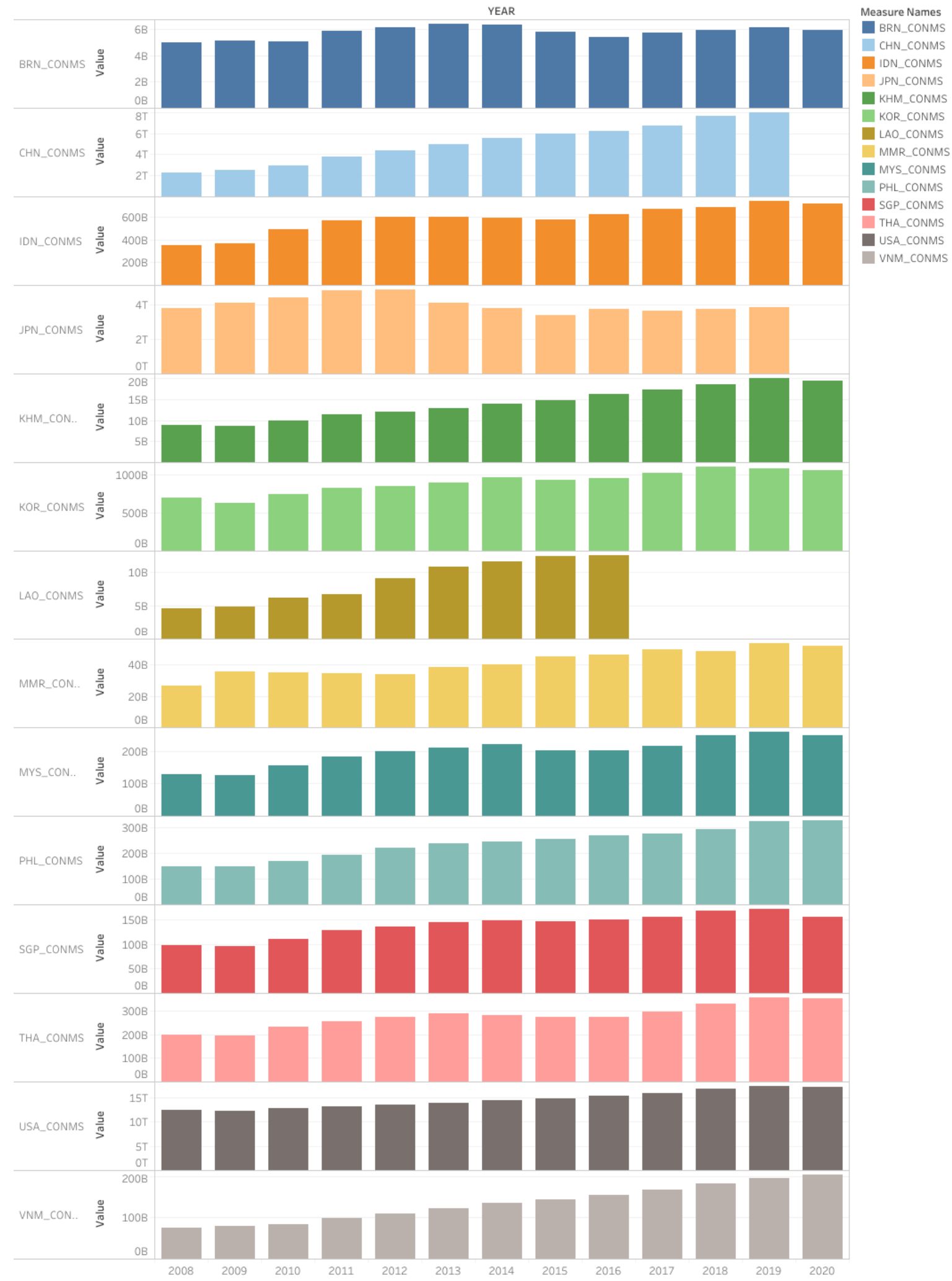

source: World Bank indicators

In the graph, we can see together, the countries with the largest consumption value are the US, China, Japan, Korea. As we can see, the advanced countries have large consumption value. 
Final consumption expenditure (\% of GDP)

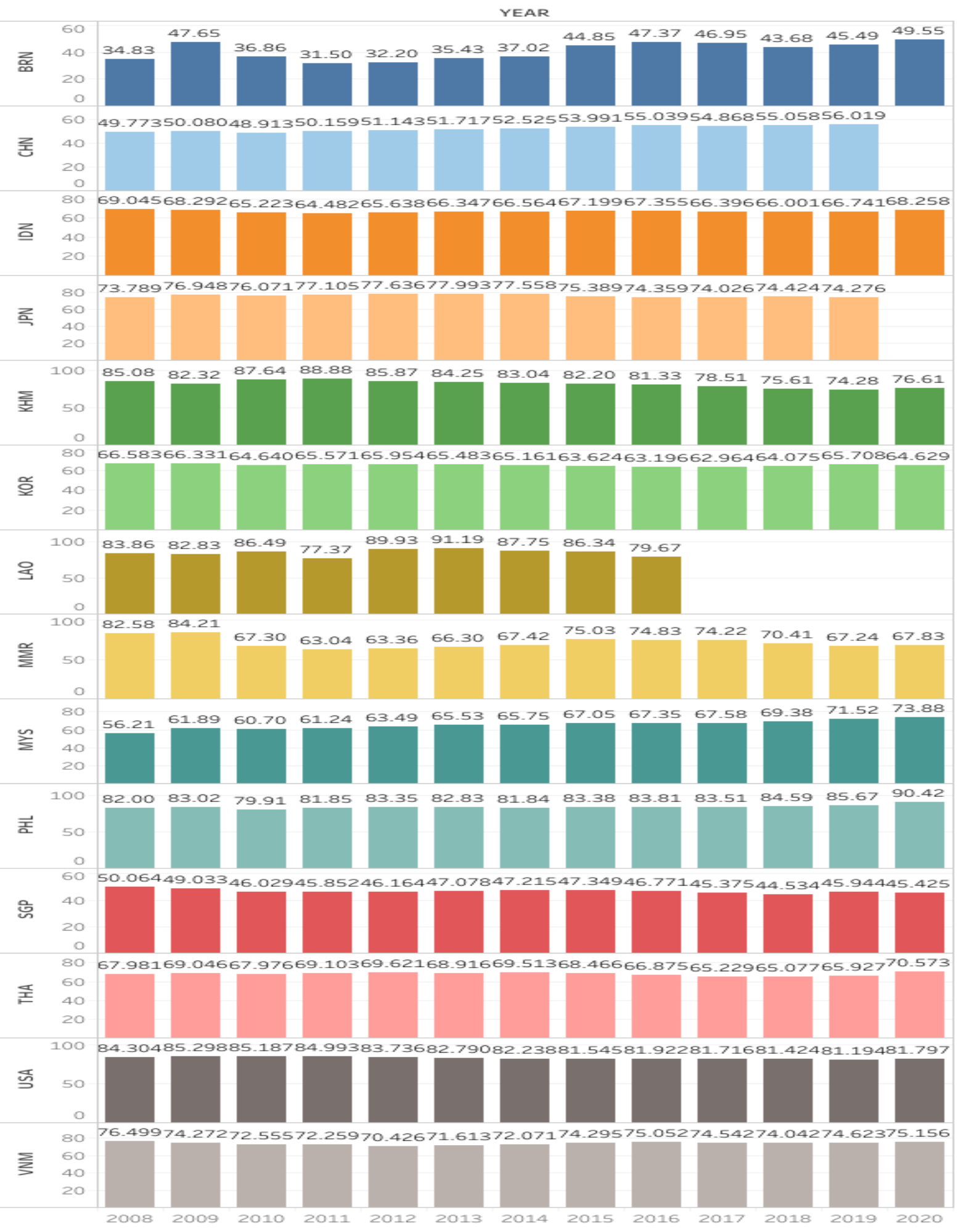

Measure Names

BRN

CHN

IDN

JPN

KOR

MiM

- MYS

PHL

SGP

THA

USA

3.78976 .94876 .07177 .10577636779937755875 .38974 .35974 .02674 .42474 .276

Based on the graph, it appears that the share consumption of each country varied depend on its economic characteristic. 
Households and NPISHs Final consumption expenditure (current US\$)

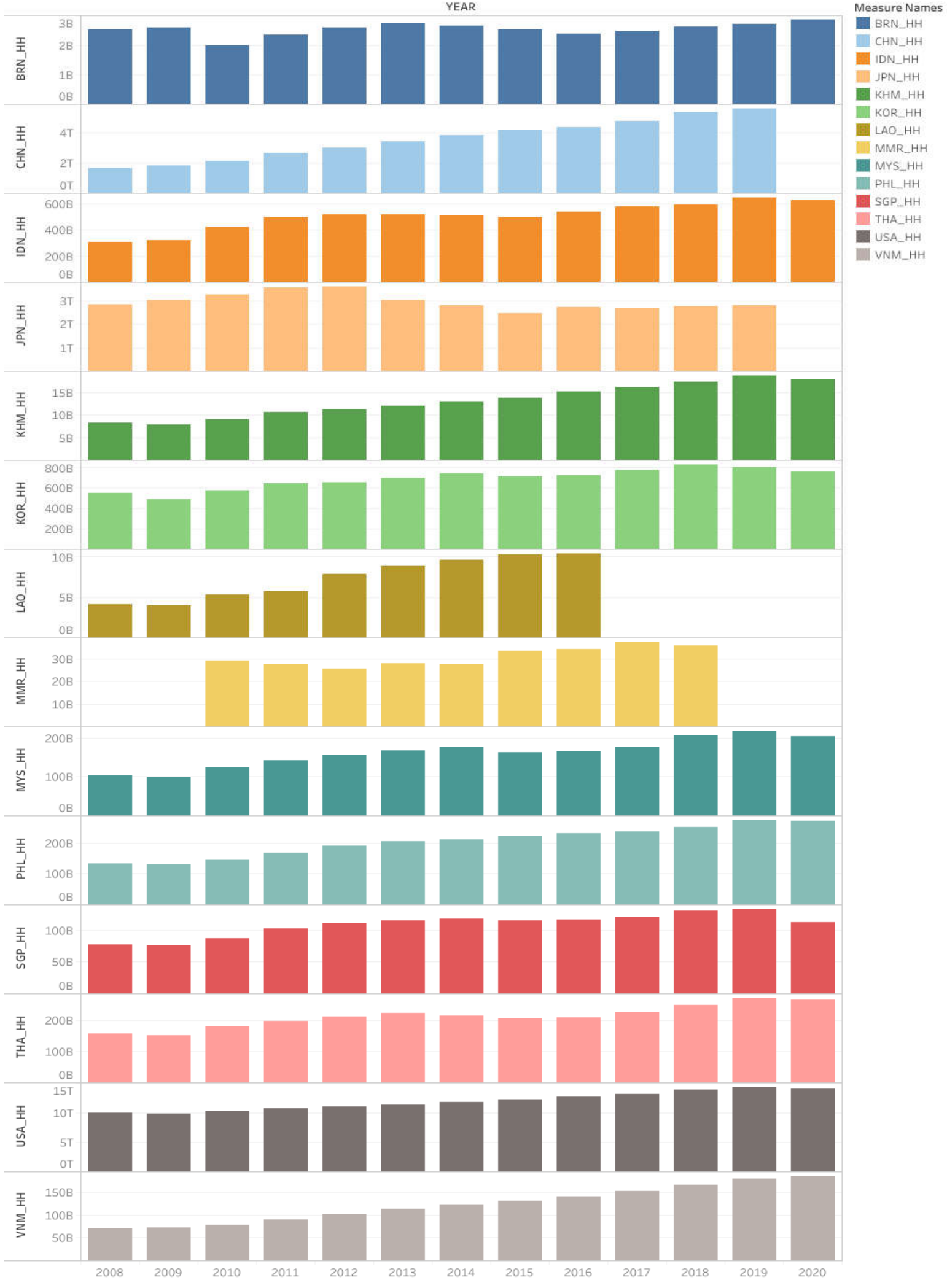

Based on the graph, we can see that the US, China, Japan, Korea have the largest value in household and NPISHs consumption expenditure. 
Index 2015=100 source: OECD,2021

US monthly CPI

USACPIALL

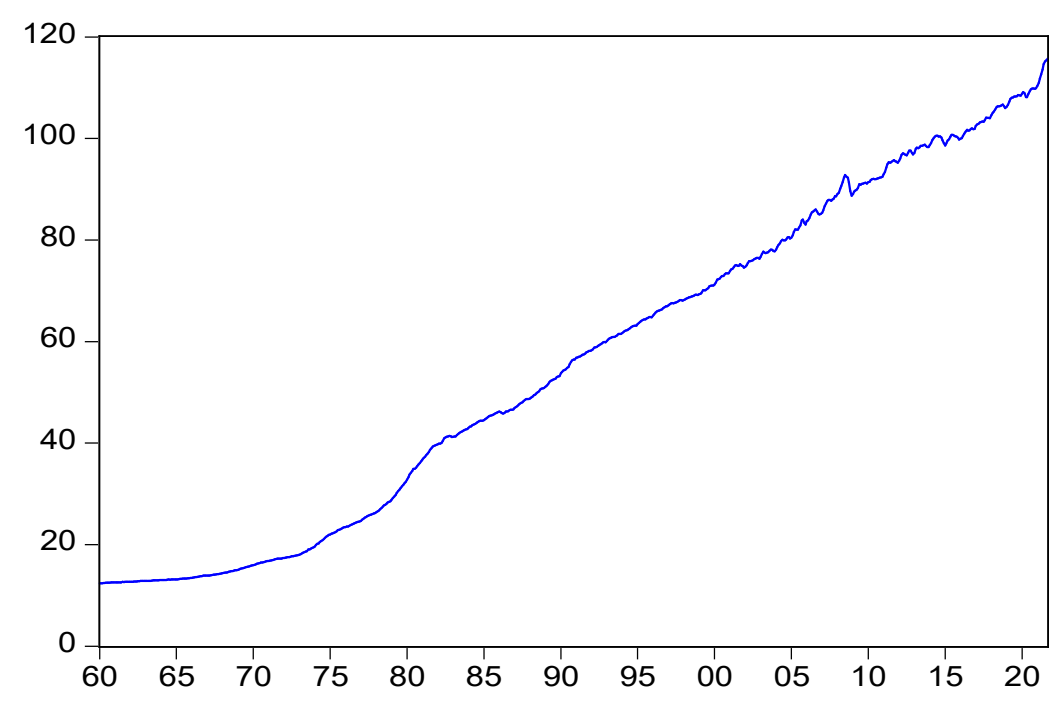

China mothly CPI

CHNCPIALL

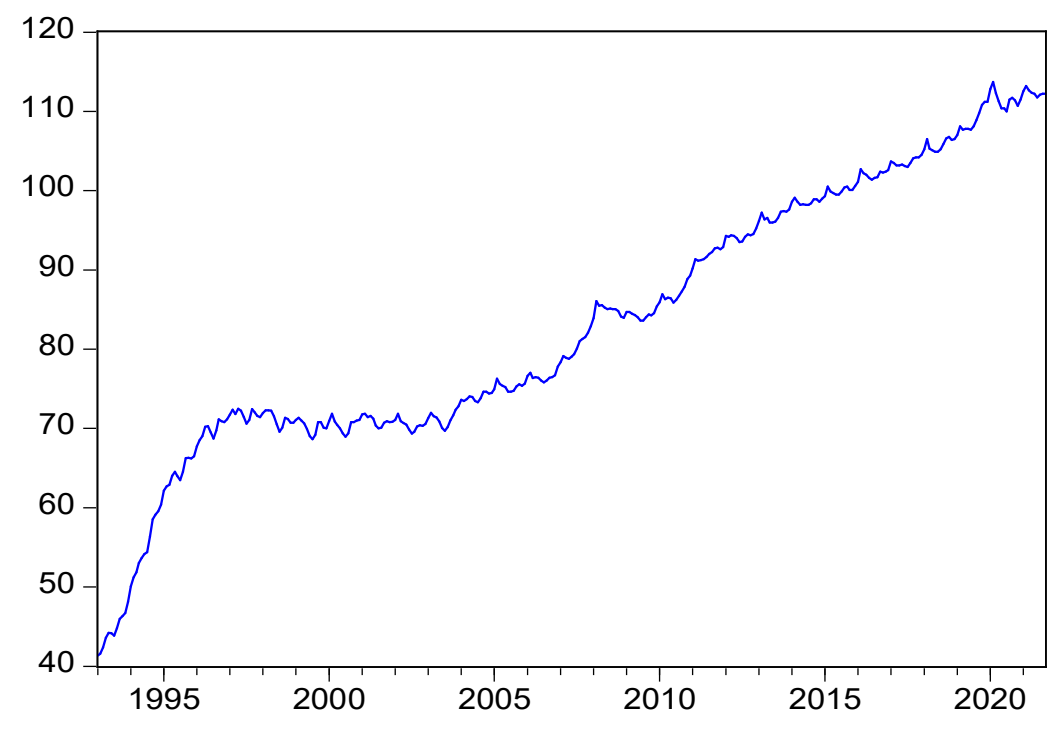


Korea monthly CPI

KORCPIALL

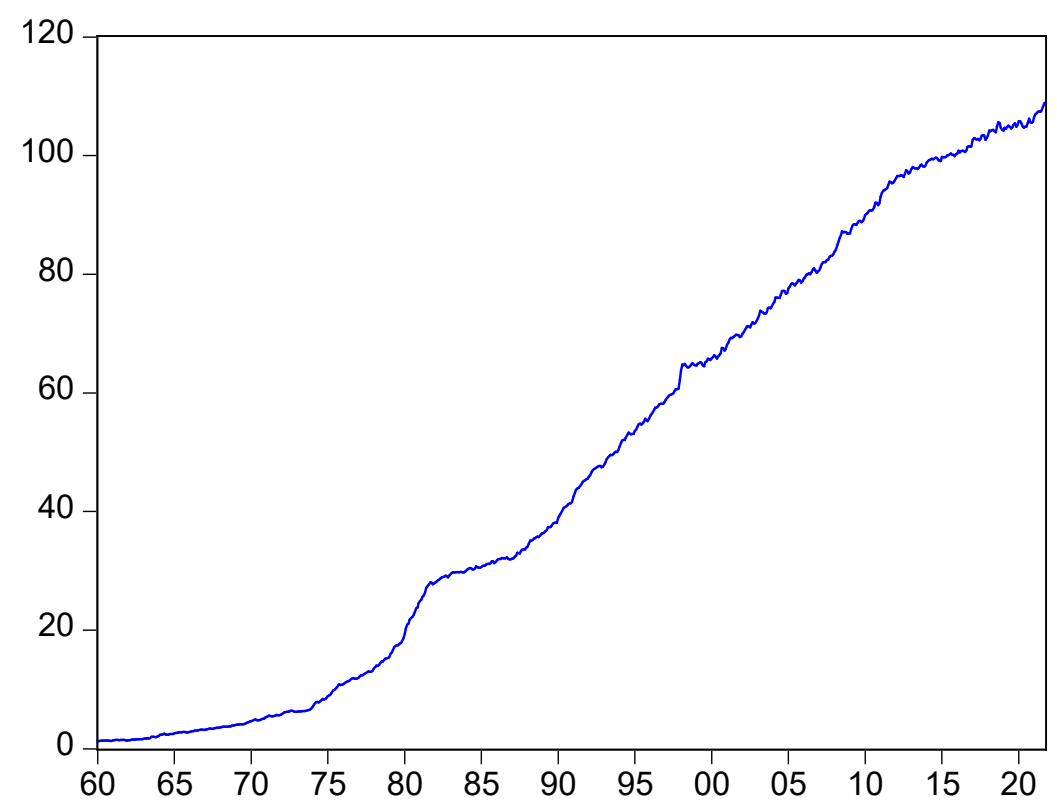

Japan monthly CPI

JPNCPIALL

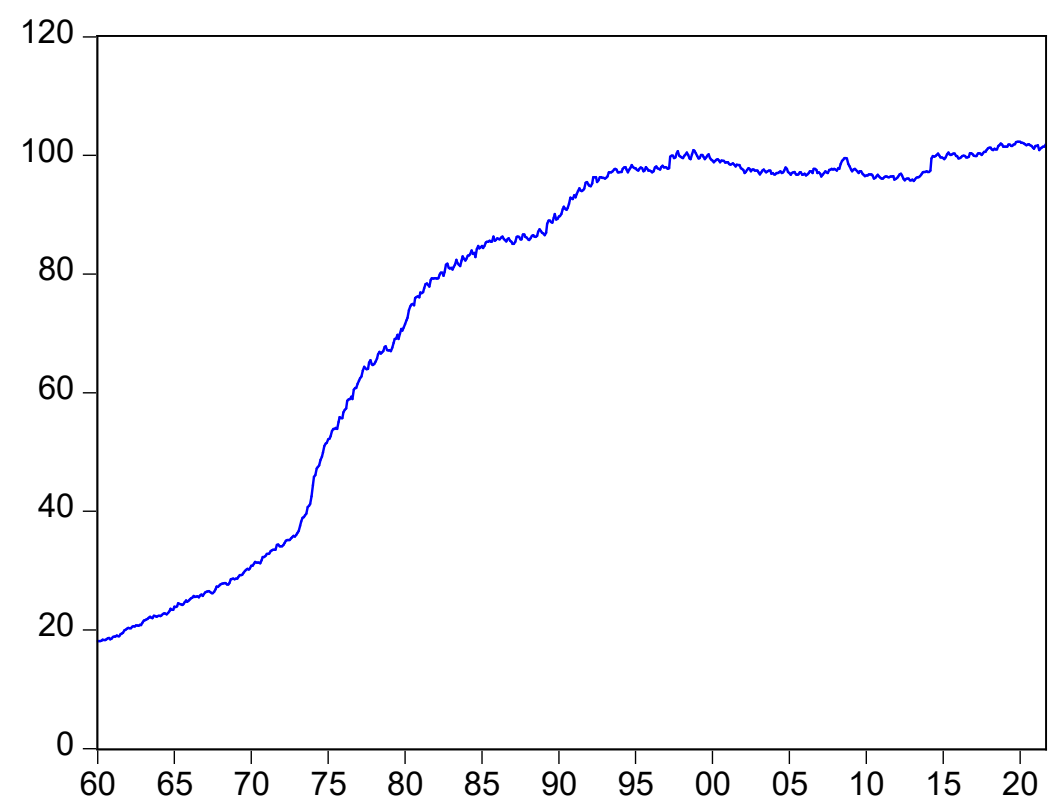


Indonesia monthly CPI

IDNCPIALL

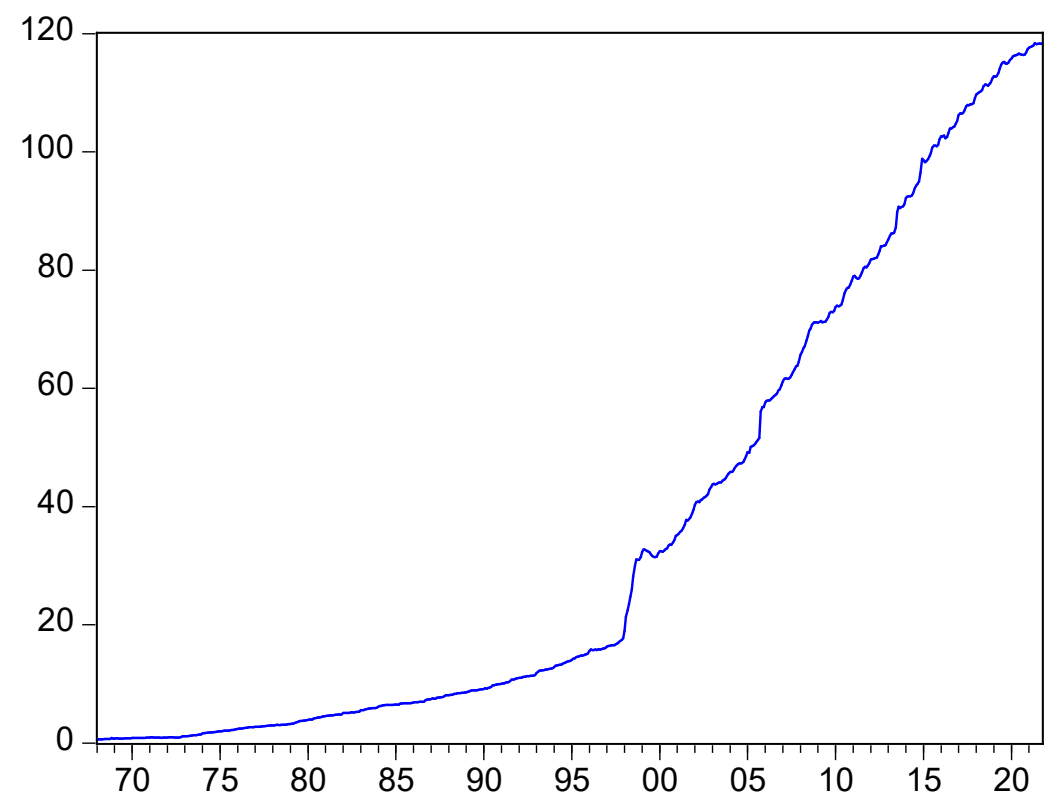

Index 2010=100, source: World Bank indicators

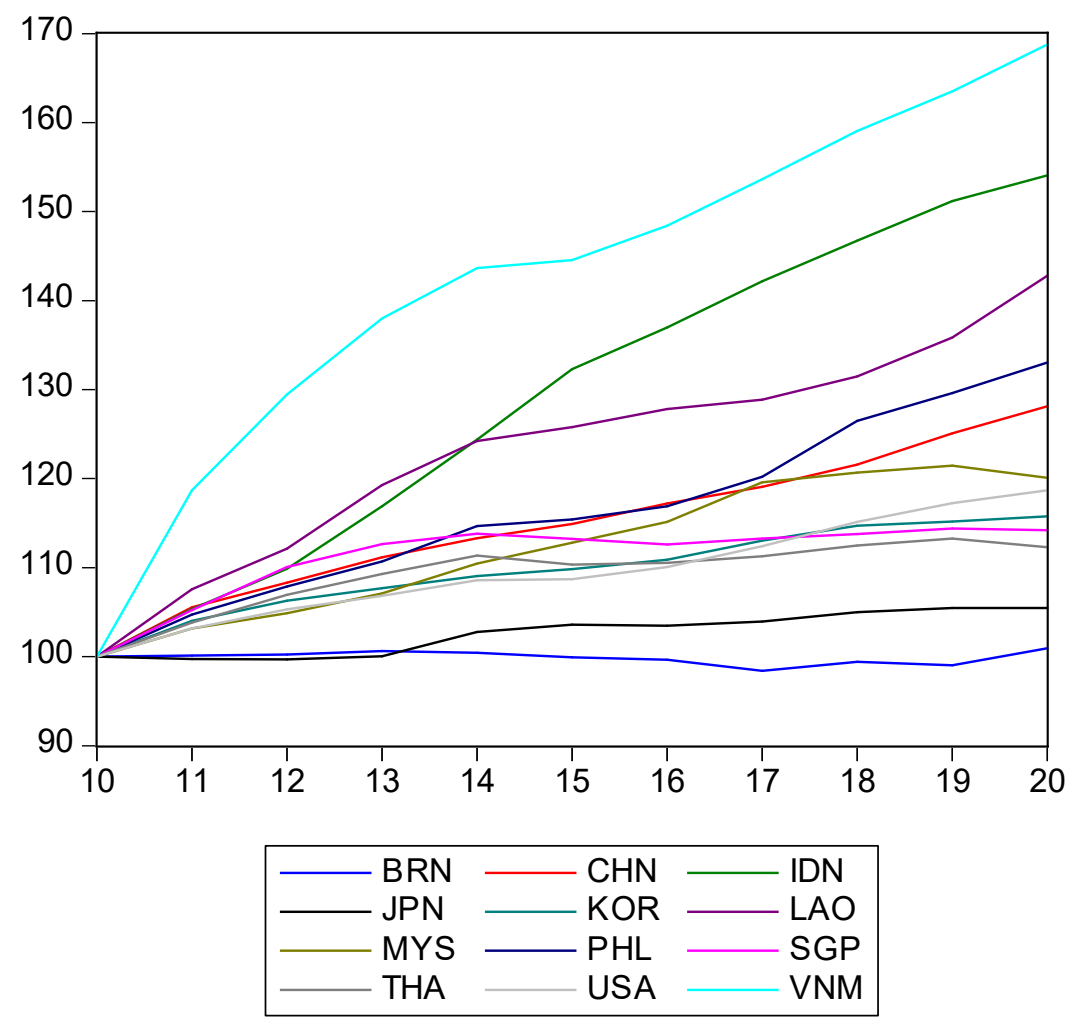




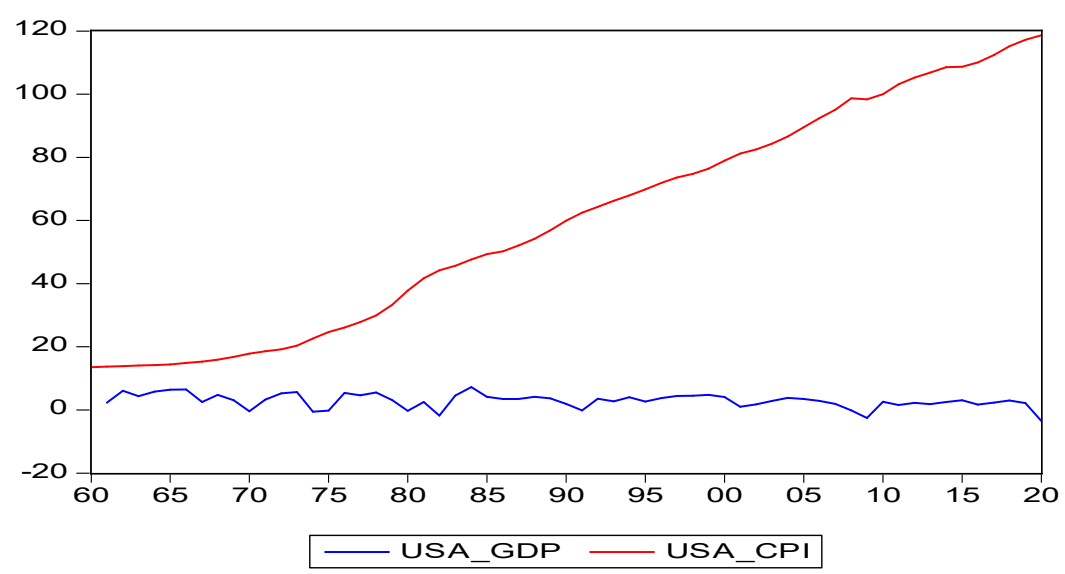

US LEAST SQUARES

Dependent Variable: USA_GDP

Method: Least Squares

Sample (adjusted): 19612020

Included observations: 60 after adjustments

\begin{tabular}{llccl}
\hline \hline Variable & Coefficient & Std. Error & t-Statistic & Prob. \\
\hline \hline USA_CPI & -0.023944 & 0.007739 & -3.093748 & 0.0030 \\
C & 4.379510 & 0.538757 & 8.128909 & 0.0000 \\
\hline \hline R-squared & 0.141647 & Mean dependent var & 2.928965 \\
Adjusted R-squared & 0.126848 & S.D. dependent var & 2.199881 \\
S.E. of regression & 2.055626 & Akaike info criterion & 4.311803 \\
Sum squared resid & 245.0847 & Schwarz criterion & 4.381615 \\
Log likelihood & -127.3541 & Hannan-Quinn criter. & 4.339110 \\
F-statistic & 9.571278 & Durbin-Watson stat & 1.468010 \\
Prob(F-statistic) & 0.003039 & & \\
\hline \hline
\end{tabular}

\section{US CUSUM}

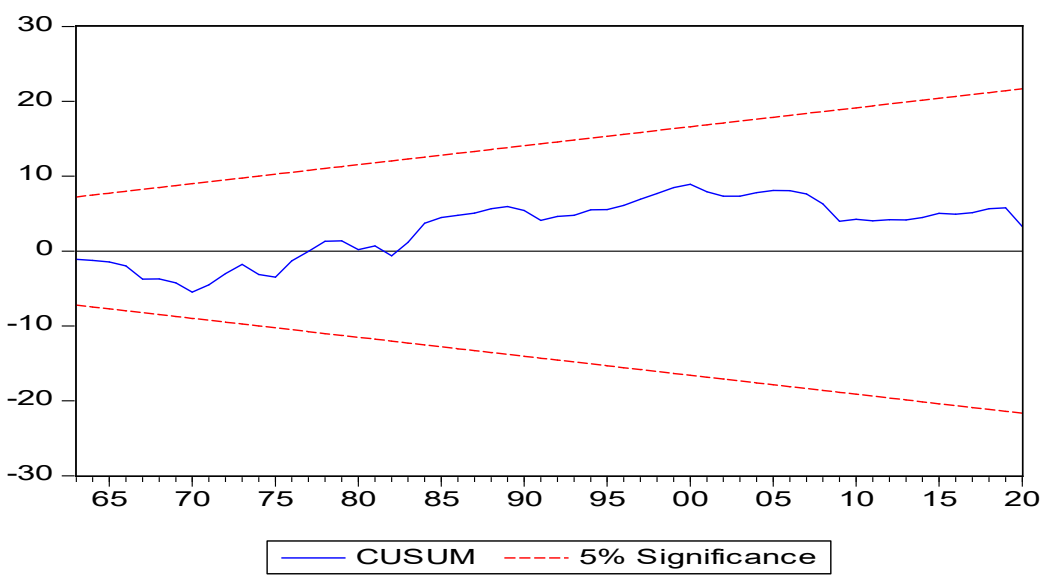


JPN CPI-GDP

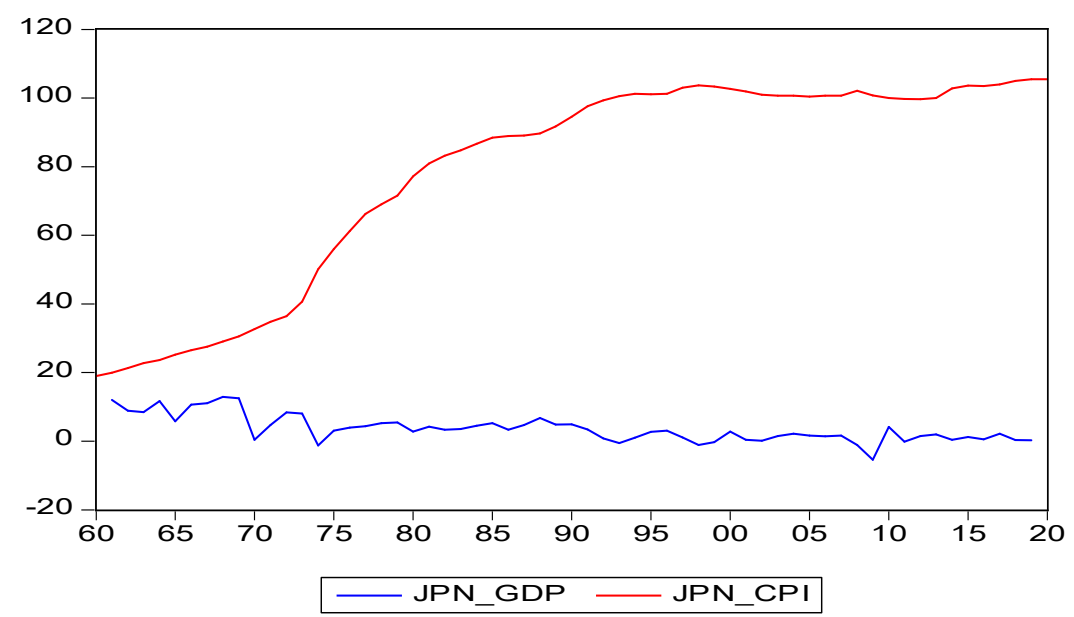

JPN- LEAST SQUARES

Dependent Variable: JPN_GDP

Method: Least Squares

Sample (adjusted): 19612019

Included observations: 59 after adjustments

\begin{tabular}{lllll}
\hline \hline Variable & Coefficient & Std. Error & t-Statistic & Prob. \\
\hline \hline JPN_CPI & -0.099485 & 0.010948 & -9.086636 & 0.0000 \\
C & 11.44091 & 0.920838 & 12.42446 & 0.0000 \\
\hline \hline R-squared & 0.591594 & Mean dependent var & 3.605451 \\
Adjusted R-squared & 0.584429 & S.D. dependent var & 3.849391 \\
S.E. of regression & 2.481503 & Akaike info criterion & 4.688916 \\
Sum squared resid & 350.9978 & Schwarz criterion & 4.759341 \\
Log likelihood & -136.3230 & Hannan-Quinn criter. & 4.716407 \\
F-statistic & 82.56695 & Durbin-Watson stat & 1.616311 \\
Prob(F-statistic) & 0.000000 & & \\
\hline \hline
\end{tabular}

JPN CUSUM CPI-GDP

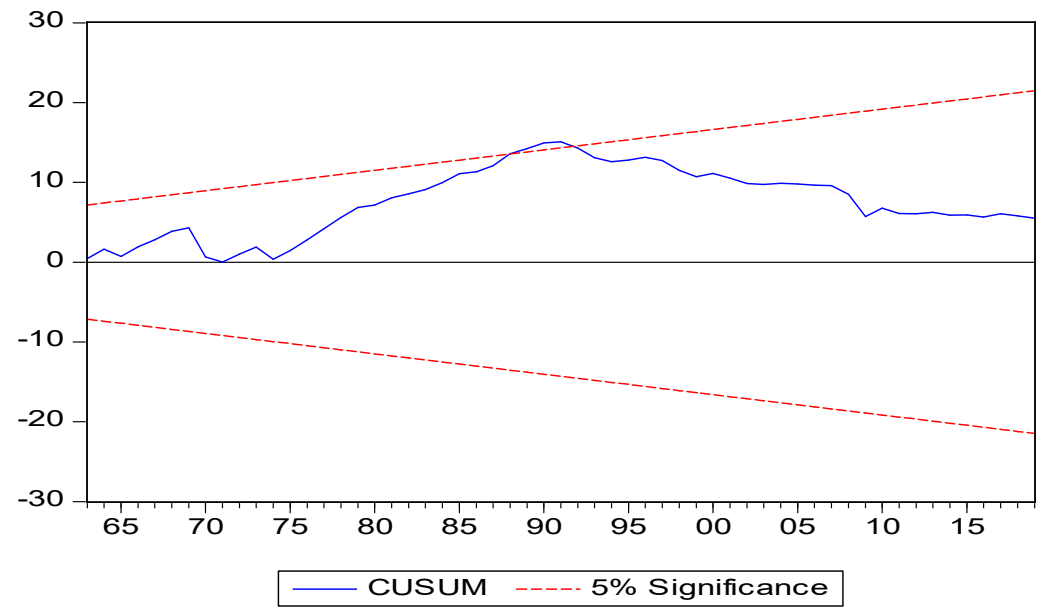


KOREA CPI-GDP

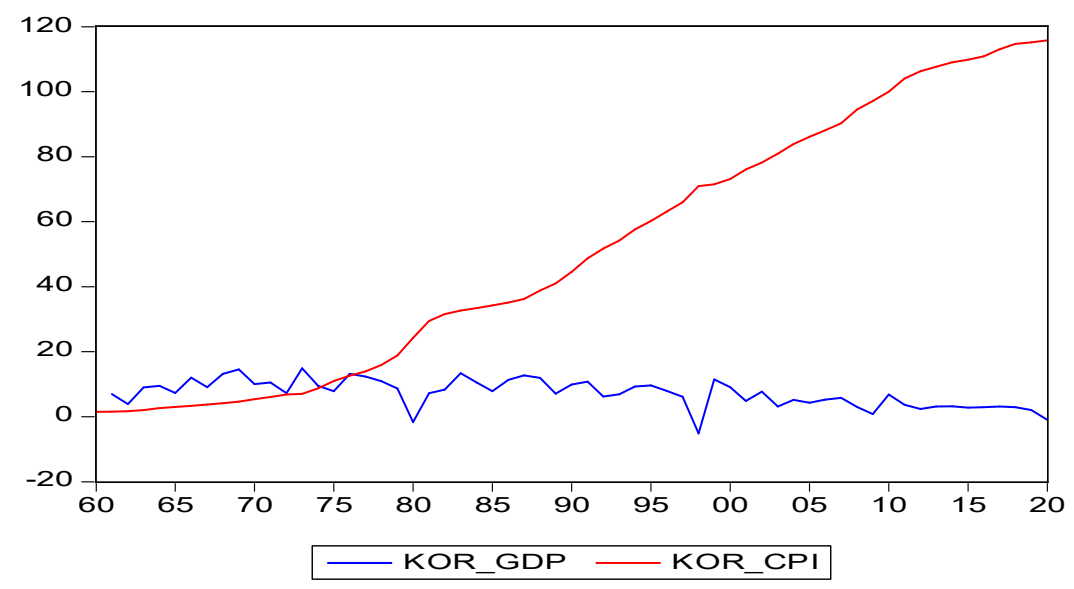

\section{KOREA LEAST SQUARES}

Dependent Variable: KOR_GDP

Method: Least Squares

Sample (adjusted): 19612020

Included observations: 60 after adjustments

\begin{tabular}{lcccc}
\hline \hline Variable & Coefficient & Std. Error & t-Statistic & Prob. \\
\hline \hline KOR_CPI & -0.069287 & 0.010574 & -6.552344 & 0.0000 \\
C & 10.82638 & 0.687307 & 15.75190 & 0.0000 \\
\hline \hline R-squared & 0.425363 & Mean dependent var & 7.230760 \\
Adjusted R-squared & 0.415455 & S.D. dependent var & 4.192708 \\
S.E. of regression & 3.205557 & Akaike info criterion & 5.200414 \\
Sum squared resid & 595.9847 & Schwarz criterion & 5.270226 \\
Log likelihood & -154.0124 & Hannan-Quinn criter. & 5.227721 \\
F-statistic & 42.93322 & Durbin-Watson stat & 1.805994 \\
Prob(F-statistic) & 0.000000 & & \\
\hline \hline
\end{tabular}

\section{KOREA CUSUM TEST}

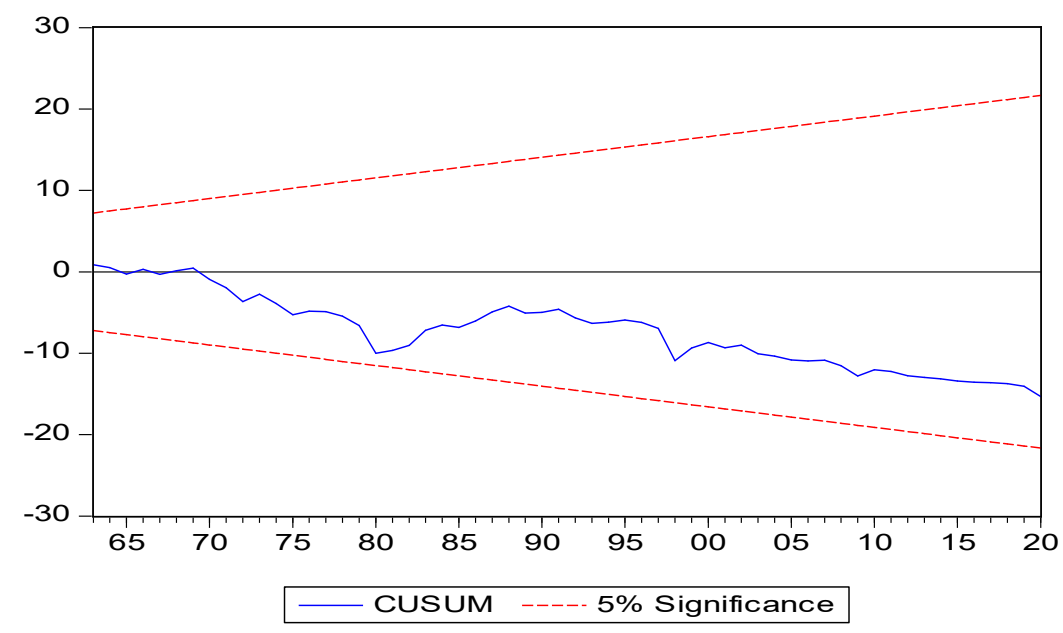




\section{SINGAPORE CPI-GDP}

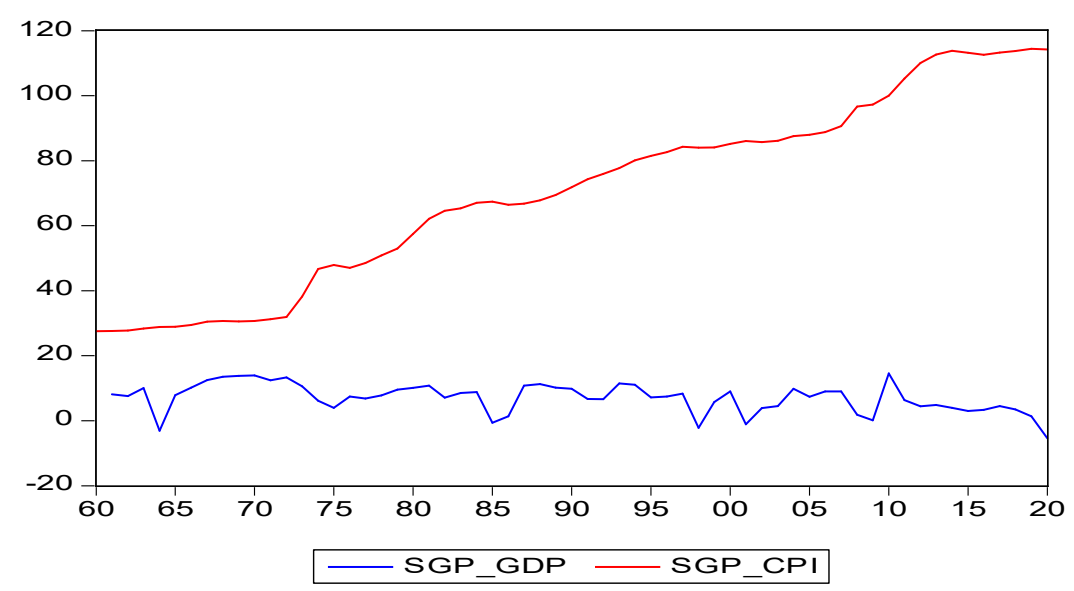

\section{SINGAPORE LEAST SQUARES}

Dependent Variable: SGP_GDP

Method: Least Squares

Sample (adjusted): 19612020

Included observations: 60 after adjustments

\begin{tabular}{lllll}
\hline \hline Variable & Coefficient & Std. Error & t-Statistic & Prob. \\
\hline \hline SGP_CPI & -0.074084 & 0.018240 & -4.061676 & 0.0001 \\
C & 12.27127 & 1.391153 & 8.820933 & 0.0000 \\
\hline \hline R-squared & 0.221447 & Mean dependent var & 7.017688 \\
Adjusted R-squared & 0.208024 & S.D. dependent var & 4.457693 \\
S.E. of regression & 3.967036 & Akaike info criterion & 5.626681 \\
Sum squared resid & 912.7677 & Schwarz criterion & 5.696492 \\
Log likelihood & -166.8004 & Hannan-Quinn criter. & 5.653988 \\
F-statistic & 16.49722 & Durbin-Watson stat & 1.458602 \\
Prob(F-statistic) & 0.000148 & & \\
\hline \hline
\end{tabular}

\section{SINGAPORE CUSUM TEST}

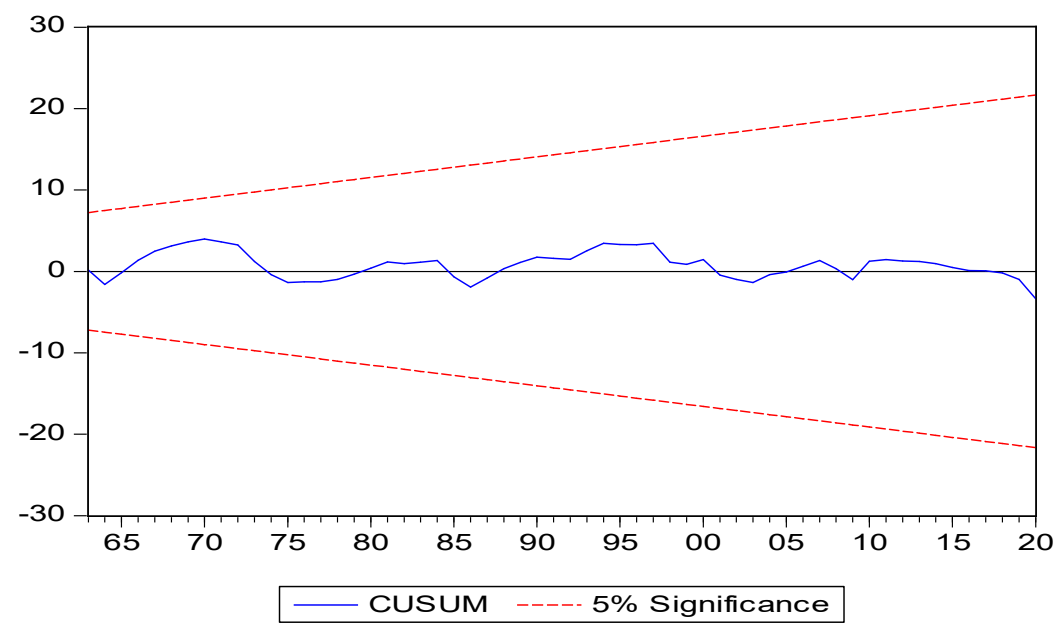


IDN CPI-GDP

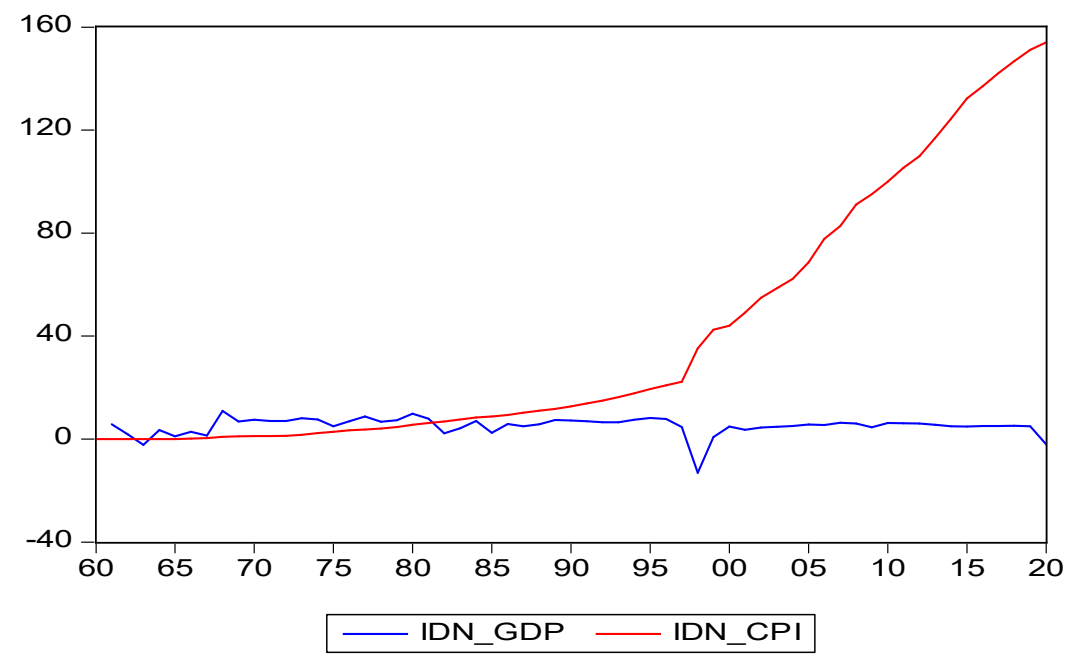

IDN- LEAST SQUARES

Dependent Variable: IDN_GDP

Method: Least Squares

Sample (adjusted): 19612020

Included observations: 60 after adjustments

\begin{tabular}{lllll}
\hline \hline Variable & Coefficient & Std. Error & t-Statistic & Prob. \\
\hline \hline IDN_CPI & -0.008644 & 0.009055 & -0.954597 & 0.3437 \\
C & 5.482294 & 0.576099 & 9.516235 & 0.0000 \\
\hline \hline R-squared & 0.015468 & Mean dependent var & 5.131592 \\
Adjusted R-squared & -0.001506 & S.D. dependent var & 3.434743 \\
S.E. of regression & 3.437329 & Akaike info criterion & 5.340032 \\
Sum squared resid & 685.2836 & Schwarz criterion & 5.409843 \\
Log likelihood & -158.2010 & Hannan-Quinn criter. & 5.367339 \\
F-statistic & 0.911255 & Durbin-Watson stat & 1.301613 \\
Prob(F-statistic) & 0.343744 & & \\
\hline \hline
\end{tabular}

IDN CUSUM CPI-GDP

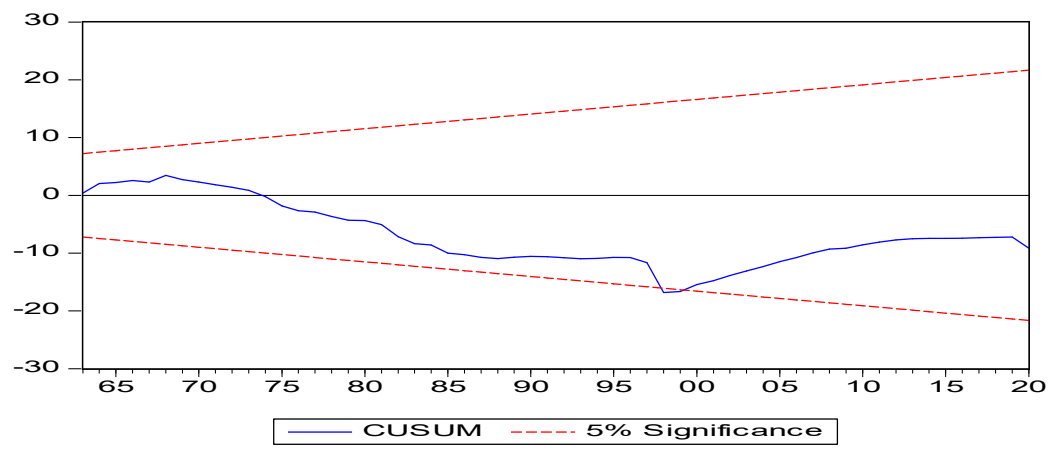




\section{Conclusion}

The results are varied in each country. On this study we learn that the GDP is influenced by the final consumption and the pattern of consumption are varied in each country.

\section{References}

Anghel, M. G., Stoica, R., Samson, T., \& Badiu, A. (2017). Analysis of the Econometric model of the correlation between GDP and final consumption. Romanian Statistical Review Supplement, 65(2), 122-129.

Asumadu-Sarkodie, S., \& Owusu, P. A. (2016). The casual nexus between child mortality rate, fertility rate, GDP, household final consumption expenditure, and food production index. Cogent Economics \& Finance, 4(1), 1191985.

Danquah, M., Moral-Benito, E., \& Ouattara, B. (2014). TFP growth and its determinants: a model averaging approach. Empirical Economics, 47(1), 227-251.

Deaton, Angus. 2020. GDP and Beyond. retrieved from: https://apps.bea.gov/scb/2020/06-june/0620-beyond-gdp-deaton.htm

Diacon, P. E., \& Maha, L. G. (2015). The relationship between income, consumption and GDP: A time series, cross-country analysis. Procedia economics and finance, 23, 1535-1543.

Shaikh, N. A., Shah, P., \& Shah, N. (2015). Empirical Estimation of GDP determinants, Household Consumption Expenditure and the Consumption Multiplier in Pakistan (1985-2011). Journal of Economics and Political Economy, 2(2), 317-330.

Perrotta, C. (1997). The preclassical theory of development: increased consumption raises productivity. History of Political Economy, 29(2), 295. 